\title{
WAŻEWSKI'S UNIVERSAL DENDRITE AS AN INVERSE LIMIT WITH ONE SET-VALUED BONDING FUNCTION
}

\author{
Iztok Banič, Matevž Črepnjak, Matej Merhar, Uroš \\ Milutinović AND Tina Sovič \\ University of Maribor and Institute of Mathematics, Physics and Mechanics, \\ Ljubljana, Slovenia
}

\begin{abstract}
We construct a family of upper semi-continuous setvalued functions $f:[0,1] \rightarrow 2^{[0,1]}$ (belonging to the class of so-called comb functions), such that for each of them the inverse limit of the inverse sequence of intervals $[0,1]$ and $f$ as the only bonding function is homeomorphic to Ważewski's universal dendrite. Among other results we also present a complete characterization of comb functions for which the inverse limits of the above type are dendrites.
\end{abstract}

\section{INTRODUCTION}

In 1923. T. Ważewski described an example of a dendrite in the plane which contains a topological copy of any dendrite ([26]). The described dendrite is now known as Ważewski's universal dendrite. In [20, p. 181] one can find a construction of Ważewski's universal dendrite using inverse limits. In particular, it is constructed as the inverse limit of an inverse sequence of planar dendrites $D_{n}$ and monotone bonding mappings $f_{n}: D_{n+1} \rightarrow D_{n}$, where dendrites $D_{n}$ are getting more and more complicated as $n$ increases, and so do the functions $f_{n}$. In this paper we construct Ważewski's universal dendrite as the inverse $\operatorname{limit} \lim _{\longleftarrow}\{[0,1], f\}_{n=1}^{\infty}$ of closed unit intervals $[0,1]$ and a single upper semi-continuous set-valued bonding function $f$. We believe that this new presentation of Ważewski's universal dendrite sheds new light on the classic dendrite and simultaniously shows the strength of the theory of the inverse systems with upper semi-continuous set-valued bonding functions.

2010 Mathematics Subject Classification. 54F50, 54C60.

Key words and phrases. Continua, inverse limits, upper semi-continuous functions, dendrites, Ważewski's universal dendrite. 


\section{Definitions and Notation}

Our definitions and notation mostly follow Nadler ([20]) and Ingram and Mahavier ([14]).

A map is a continuous function. For $i=1,2, \pi_{i}:[0,1] \times[0,1] \rightarrow[0,1]$ denotes the $i$-th projection from $[0,1] \times[0,1]$ onto the $i$-th factor, and for any positive integer $i, p_{i}: \Pi_{n=1}^{\infty}[0,1] \rightarrow[0,1]$ denotes the $i$-th projection from $\Pi_{n=1}^{\infty}[0,1]$ onto the $i$-th factor.

A continuum is a nonempty, compact and connected metric space. A Peano continuum is a locally connected continuum.

A dendrite is a Peano continuum which contains no simple closed curve.

Let $D$ be a dendrite, $b \in D$, and $\beta$ a cardinal number. We say that $b$ is of order less than or equal to $\beta$ in $D$, written $\operatorname{ord}(b, D) \leq \beta$, provided that for each open neighborhood $U$ of $b$ in $D$, there is an open neighborhood $V$ of $b$ in $D$, such that $b \in V \subseteq U$ and $|\operatorname{Bd}(V)| \leq \beta$. We say that $b$ is of order $\beta$, ord $(b, D)=\beta$, provided that $\operatorname{ord}(b, D) \leq \beta$ and $\operatorname{ord}(b, D) \not \alpha$ for any cardinal number $\alpha<\beta$.

Points of order 1 in a dendrite $D$ are called end points of $D$, the set of all end points of $D$ is denoted by $E(D)$. Points of order $n>2$ are called ramification points and the set of all ramification points of $D$ is denoted by $R(D)$.

A free arc in a dendrite $D$ is an arc such that all its points but its end points are of order 2 in $D$. In particular, a maximal free arc in a dendrite $D$ is an $\operatorname{arc} A$ with end points $x$ and $y$ in $D$ such that $A \cap(E(D) \cup R(D))=\{x, y\}$.

A continuum $S$ is a star if there is a point $c \in S$ such that $S$ can be presented as the countable union $S=\bigcup_{n=1}^{\infty} B_{n}$ of arcs $B_{n}$, each having $c$ as an end point and satisfying $\lim _{n \rightarrow \infty} \operatorname{diam}\left(B_{n}\right)=0$, such that $B_{n} \cap B_{m}=\{c\}$ when $m \neq n$. The point $c$ is uniquely determined and is called the center of $S$. The $\operatorname{arcs} B_{n}$ are called beams of $S$.

Let $D_{1}$ be a star in a compact metric space $X$. Let $c_{A} \notin R\left(D_{1}\right)$ denote a point in the maximal free $\operatorname{arc} A$, for each maximal free $\operatorname{arc} A$ of $D_{1}$ (here maximal free arcs are precisely the beams of $\left.D_{1}\right)$. Let $C_{1}=\left\{x_{1}, x_{2}, x_{3}, \ldots\right\}$ be any subset of the set $\left\{c_{A} \mid A\right.$ is a maximal free arc in $\left.D_{1}\right\}$. For each positive integer $i$, form a star $S_{i}$ in $X$ with the center $x_{i}$ and otherwise disjoint from $D_{1}$, making sure that $S_{i} \cap S_{j} \neq \emptyset$ only when $i=j$ and that $\lim _{i \rightarrow \infty} \operatorname{diam}\left(S_{i}\right)=0$. Let $D_{2}=D_{1} \cup\left(\bigcup_{i=1}^{\infty} S_{i}\right)$. Next define $D_{3}$ in a similar manner. Let $c_{A} \notin R\left(D_{2}\right)$ denote a point in the maximal free arc $A$ in $D_{2}$, for each maximal free arc $A$ of $D_{2}$. Let $C_{2}=\left\{x_{1}, x_{2}, x_{3}, \ldots\right\}$ be any subset of the set $\left\{c_{A} \mid A\right.$ is a maximal free arc in $\left.D_{2}\right\}$. For each positive integer $i$, form a star $S_{i}$ in $X$ with the center $x_{i}$ and otherwise disjoint from $D_{2}$, making sure that $S_{i} \cap S_{j} \neq \emptyset$ only when $i=j$ and that $\lim _{i \rightarrow \infty} \operatorname{diam}\left(S_{i}\right)=0$. Let 
$D_{3}=D_{2} \cup\left(\bigcup_{i=1}^{\infty} S_{i}\right)$. Continuing in this manner, we obtain a continuum $D_{n}$ for each positive integer $n$. The following theorem (already implicitly used in the above inductive construction) is a well-known fact, see [20] for details.

Theorem 2.1. For each positive integer $n, D_{n}$ is a dendrite.

The construction of the continuum, homeomorphic to Ważewski's universal dendrite in $[20$, p. 181] uses the above mentioned construction of a chain of dendrites $D_{1} \subseteq D_{2} \subseteq D_{3} \subseteq \ldots$, then defines certain bonding maps $f_{n}: D_{n+1} \rightarrow D_{n}$, and then finally obtains Ważewski's universal dendrite as $\lim _{\longleftarrow}\left\{D_{k}, f_{k}\right\}_{k=1}^{\infty}$.

Finally we state a result that is characterizing Ważewski's universal dendrite that will be needed in Section 4 .

Theorem 2.2. For any dendrite D, D is homeomorphic to Ważewski's universal dendrite if and only if its set of ramification points is dense in $D$ and each of its ramification points is of infinite order.

Proof. [7, p. 169], [26, p. 123]

If $(X, d)$ is a compact metric space, then $2^{X}$ denotes the set of all nonempty closed subsets of $X$. Let for each $\varepsilon>0$ and each $A \in 2^{X}$

$$
N_{d}(\varepsilon, A)=\{x \in X \mid d(x, a)<\varepsilon \text { for some } a \in A\} .
$$

The set $2^{X}$ will be always equipped with the Hausdorff metric $H_{d}$, which is defined by

$$
H_{d}(H, K)=\inf \left\{\varepsilon>0 \mid H \subseteq N_{d}(\varepsilon, K), K \subseteq N_{d}(\varepsilon, H)\right\},
$$

for $H, K \in 2^{X}$. Then $\left(2^{X}, H_{d}\right)$ is a metric space, called the hyperspace of the space $(X, d)$. For more details see $[13,20]$.

When we say that $f$ is a set-valued function from $X$ to $Y$, we mean that $f$ is a single-valued function from $X$ to $2^{Y}$, i.e., $f: X \rightarrow 2^{Y}$. By a slight abuse of notation and terminology we will also say that function $f: X \rightarrow 2^{Y}$ is set-valued (without explicitly mentioning "from $X$ to $Y$ ").

A function $f: X \rightarrow 2^{Y}$ is surjective set-valued function from $X$ to $Y$ if for each $y \in Y$ there is an $x \in X$, such that $y \in f(x)$.

The graph $\Gamma(f)$ of a set-valued function $f: X \rightarrow 2^{Y}$ is the set of all points $(x, y) \in X \times Y$ such that $y \in f(x)$.

A function $f: X \rightarrow 2^{Y}$, where $X$ and $Y$ are compact metric spaces, is upper semi-continuous set-valued function from $X$ to $Y$ (abbreviated u.s.c.) if for each open set $V \subseteq Y$ the set $\{x \in X \mid f(x) \subseteq V\}$ is an open set in $X$.

The following is a well-known characterization of u.s.c. functions between metric compacta (see [14, p. 120, Theorem 2.1]).

TheOREm 2.3. Let $X$ and $Y$ be compact metric spaces and $f: X \rightarrow 2^{Y}$ a set-valued function. Then $f$ is u.s.c. if and only if its graph $\Gamma(f)$ is closed in $X \times Y$. 
An inverse sequence of compact metric spaces $X_{k}$ with u.s.c. bonding functions $f_{k}$ is a sequence $\left\{X_{k}, f_{k}\right\}_{k=1}^{\infty}$, where $f_{k}: X_{k+1} \rightarrow 2^{X_{k}}$ for each $k$.

The inverse limit of an inverse sequence $\left\{X_{k}, f_{k}\right\}_{k=1}^{\infty}$ with u.s.c. bonding functions is defined to be the subspace of the product space $\prod_{k=1}^{\infty} X_{k}$ of all $x=\left(x_{1}, x_{2}, x_{3}, \ldots\right) \in \prod_{k=1}^{\infty} X_{k}$, such that $x_{k} \in f_{k}\left(x_{k+1}\right)$ for each $k$. The inverse limit of $\left\{X_{k}, f_{k}\right\}_{k=1}^{\infty}$ is denoted by $\lim _{\longleftarrow}\left\{X_{k}, f_{k}\right\}_{k=1}^{\infty}$.

Note that each inverse sequence $\left\{X_{k}, f_{k}\right\}_{k=1}^{\infty}$ with continuous singlevalued bonding functions can be interpreted as an inverse sequence with u.s.c. set-valued bonding functions and that the inverse limits obtained according to both interpretations coincide. Therefore we do not specially emphasize the status of bonding functions in inverse sequences we deal with.

The notion of the inverse limit of an inverse sequence with u.s.c. bonding functions was introduced by Mahavier in [18] and Ingram and Mahavier in [14]. Since the introduction of such inverse limits, there has been much interest in the subject and many papers appeared $([1-6,8,9,11,12,15,16,21-25])$.

The most important case in the present paper is the case when for each $k, X_{k}=[0,1]$ and $f_{k}=f$ for some $f:[0,1] \rightarrow 2^{[0,1]}$. In such case the inverse limit will be denoted by $\lim _{\longleftarrow}\{[0,1], f\}_{k=1}^{\infty}$.

On the product space $\prod_{n=1}^{\infty} X_{n}$, where $\left(X_{n}, d_{n}\right)$ is a compact metric space for each $n$, and the set of all diameters of $\left(X_{n}, d_{n}\right)$ is majorized by 1 , we use the metric

$$
D(x, y)=\sup _{n \in\{1,2,3, \ldots\}}\left\{\frac{d_{n}\left(x_{n}, y_{n}\right)}{2^{n}}\right\}
$$

where $x=\left(x_{1}, x_{2}, x_{3}, \ldots\right), y=\left(y_{1}, y_{2}, y_{3}, \ldots\right)$. It is well known that the metric $D$ induces the product topology ([10, p. 190]).

\section{The COMB FunCtions}

Let $A \subseteq[0,1] \times[0,1]$ be defined by

$$
A=\{(t, t) \in[0,1] \times[0,1] \mid t \in[0,1]\} .
$$

For any positive integer $n$, let $\left\{\left(a_{i}, b_{i}\right)\right\}_{i=1}^{n}$ be a finite sequence in $[0,1] \times[0,1]$, such that $a_{i}<b_{i}$ for each $i=1,2,3, \ldots, n$ and $a_{i} \neq a_{j}$ whenever $i \neq j$. Next denote by $A\left(a_{i}, b_{i}\right)_{i=1}^{n}$ the union

$$
A\left(a_{i}, b_{i}\right)_{i=1}^{n}=\bigcup_{i=1}^{n}\left(\left[a_{i}, b_{i}\right] \times\left\{a_{i}\right\}\right) \subseteq[0,1] \times[0,1] .
$$

Then

$$
G\left(a_{i}, b_{i}\right)_{i=1}^{n}=A \cup A\left(a_{i}, b_{i}\right)_{i=1}^{n}
$$

is closed in $[0,1] \times[0,1]$, since it is a union of finitely many closed arcs. Furthermore $\pi_{1}\left(G\left(a_{i}, b_{i}\right)_{i=1}^{n}\right)=\pi_{2}\left(G\left(a_{i}, b_{i}\right)_{i=1}^{n}\right)=[0,1]$. Therefore by 
Theorem 2.3 there is a surjective u.s.c. function $f_{\left(a_{i}, b_{i}\right)_{i=1}^{n}}:[0,1] \rightarrow 2^{[0,1]}$ such that its graph $\Gamma\left(f_{\left(a_{i}, b_{i}\right)_{i=1}^{n}}\right)$ equals to $G\left(a_{i}, b_{i}\right)_{i=1}^{n}$.

Definition 3.1. Let $n$ be a positive integer and $\left\{\left(a_{i}, b_{i}\right)\right\}_{i=1}^{n}$ be a subset of $[0,1] \times[0,1]$, such that $0<a_{i}<b_{i}$ for each $i=1,2,3, \ldots, n$ and $a_{i} \neq a_{j}$ whenever $i \neq j$. Then $f:[0,1] \rightarrow 2^{[0,1]}$ is called an $n$-comb function with respect to $\left\{\left(a_{i}, b_{i}\right)\right\}_{i=1}^{n}$, if $f=f_{\left(a_{i}, b_{i}\right)_{i=1}^{n}}$.

We also say that $f:[0,1] \rightarrow 2^{[0,1]}$ is an $n$-comb function, if $f$ is an $n$-comb function with respect to some $\left\{\left(a_{i}, b_{i}\right)\right\}_{i=1}^{n}$.

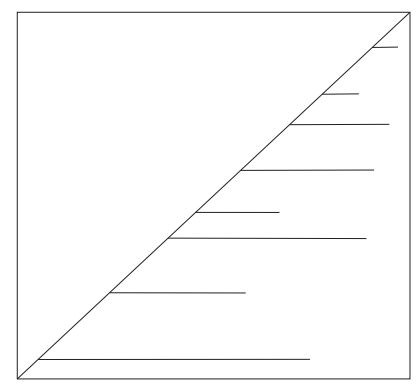

FiguRE 1. The graph of an 8-comb function

It is not necessary to eliminate the possibility $a_{i}=0$ for some $i$ (all the proofs in the paper would go through also in such case), but we have chosen to do so in order to reduce the number of cases that must be examined in the proofs and since the main result can be obtained with this restriction in place.

Definition 3.2. Let for each $j, i_{j}$ be a nonnegative integer. We use

$$
\left(a_{1}^{i_{1}}, a_{2}^{i_{2}}, a_{3}^{i_{3}}, \ldots\right)
$$

to denote the point $(\underbrace{a_{1}, a_{1}, \ldots, a_{1}}_{i_{1}}, \underbrace{a_{2}, a_{2}, \ldots, a_{2}}_{i_{2}}, \ldots)$ and

$$
\left(a_{1}^{i_{1}}, a_{2}^{i_{2}}, a_{3}^{i_{3}}, \ldots, a_{j}^{i_{j}}, t^{\infty}\right)
$$

to denote the point $(\underbrace{a_{1}, a_{1}, \ldots, a_{1}}_{i_{1}}, \underbrace{a_{2}, a_{2}, \ldots, a_{2}}_{i_{2}}, \ldots, \underbrace{a_{j}, a_{j}, \ldots, a_{j}}_{i_{j}}, t, t, t, \ldots)$.

ExAMPLE 3.3. Let $f$ be a 1 -comb function with respect to $\left\{\left(a_{i}, b_{i}\right)\right\}_{i=1}^{1}$. Then $x \in \lim _{\longleftarrow}\{[0,1], f\}_{k=1}^{\infty}$ if and only if

1. either $x=\left(t^{\infty}\right)$ for a $t \in[0,1]$ or

2. there is a positive integer $n$ such that $x=\left(a_{1}^{n}, t^{\infty}\right)$ for a $t \in\left(a_{1}, b_{1}\right]$. 
Therefore $\lim _{\lfloor}\{[0,1], f\}_{k=1}^{\infty}$ is the star with the center $\left(a_{1}^{\infty}\right)$ and beams $B_{0}=$ $\left\{\left(t^{\infty}\right) \mid t \in\left[0, a_{1}\right]\right\}, B_{0}^{\prime}=\left\{\left(t^{\infty}\right) \mid t \in\left[a_{1}, 1\right]\right\}$ and $B_{n}=\left\{\left(a_{1}^{n}, t^{\infty}\right) \mid t \in\left[a_{1}, b_{1}\right]\right\}$, $n=1,2,3, \ldots$
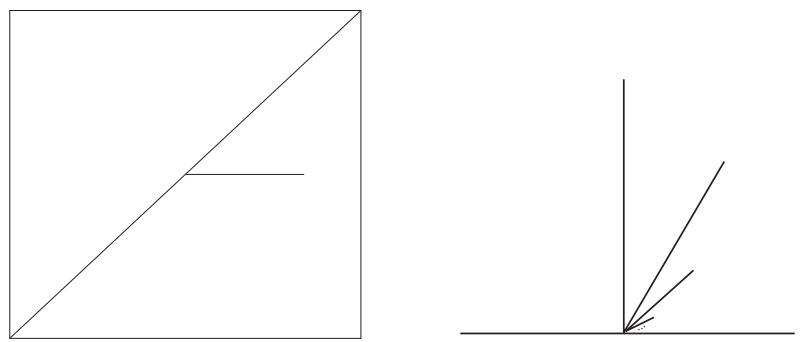

Figure 2. The graph of a 1-comb function and its inverse limit

EXAMPLE 3.4. Let $f$ be a 2-comb function with respect to $\left\{\left(a_{i}, b_{i}\right)\right\}_{i=1}^{2}$, where $a_{1}<a_{2}$. We distinguish the following two cases:

1. $b_{1}<a_{2}$

Then $x \in \lim _{\longleftarrow}\{[0,1], f\}_{k=1}^{\infty}$ if and only if

(a) either $x=\left(t^{\infty}\right)$ for a $t \in[0,1]$ or

(b) there is a positive integer $n$ such that $x=\left(a_{1}^{n}, t^{\infty}\right)$ for a $t \in$ $\left(a_{1}, b_{1}\right]$ or

(c) there is a positive integer $n$ such that $x=\left(a_{2}^{n}, t^{\infty}\right)$ for a $t \in$ $\left(a_{2}, b_{2}\right]$.

Therefore $\lim _{\longleftarrow}\{[0,1], f\}_{k=1}^{\infty}$ is the union of two stars. The star $S$ with the center $\left(a_{1}^{\infty}\right)$ and beams $B_{0}=\left\{\left(t^{\infty}\right) \mid t \in\left[0, a_{1}\right]\right\}, B_{0}^{\prime}=$ $\left\{\left(t^{\infty}\right) \mid t \in\left[a_{1}, 1\right]\right\}$ and $B_{n}=\left\{\left(a_{1}^{n}, t^{\infty}\right) \mid t \in\left[a_{1}, b_{1}\right]\right\}, n=1,2,3, \ldots$, and the star $S_{0}$ with the center $\left(a_{2}^{\infty}\right)$ and beams $C_{n}=\left\{\left(a_{2}^{n}, t^{\infty}\right) \mid t \in\right.$ $\left.\left[a_{2}, b_{2}\right]\right\}, n=1,2,3, \ldots$
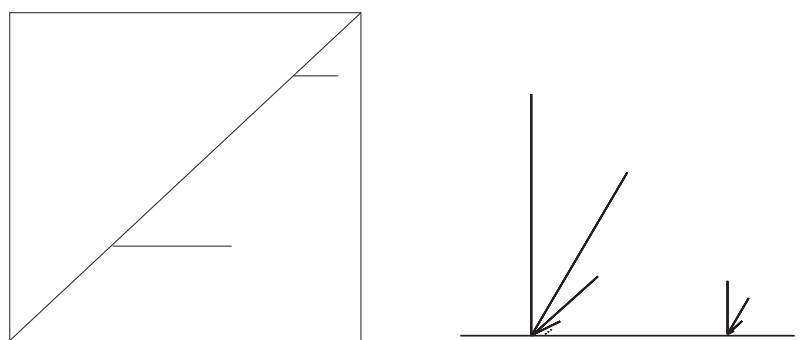

Figure 3 . The graph of a 2 -comb function and its inverse limit, $b_{1}<a_{2}$ 
2. $b_{1} \geq a_{2}$

Then $x \in \lim \{[0,1], f\}_{k=1}^{\infty}$ if and only if

(a) either $x=\left(t^{\infty}\right)$ for a $t \in[0,1]$ or

(b) there is a positive integer $n$ such that $x=\left(a_{1}^{n}, t^{\infty}\right)$ for a $t \in$ $\left(a_{1}, b_{1}\right]$ or

(c) there is a positive integer $n$ such that $x=\left(a_{2}^{n}, t^{\infty}\right)$ for a $t \in$ $\left(a_{2}, b_{2}\right]$ or

(d) there are positive integers $n$ and $m$ such that $x=\left(a_{1}^{n}, a_{2}^{m}, t^{\infty}\right)$ for a $t \in\left(a_{2}, b_{2}\right]$.

Therefore $\lim _{\longleftarrow}\{[0,1], f\}_{k=1}^{\infty}$ is the union of countable many stars. The star $S$ with the center $\left(a_{1}^{\infty}\right)$ and beams $B_{0}=\left\{\left(t^{\infty}\right) \mid t \in\left[0, a_{1}\right]\right\}$, $B_{0}^{\prime}=\left\{\left(t^{\infty}\right) \mid t \in\left[a_{1}, 1\right]\right\}$ and $B_{n}=\left\{\left(a_{1}^{n}, t^{\infty}\right) \mid t \in\left[a_{1}, b_{1}\right]\right\}, n=$ $1,2,3, \ldots$, the star $S_{0}$ with the center $\left(a_{2}^{\infty}\right)$ and beams

$$
C_{n}=\left\{\left(a_{2}^{n}, t^{\infty}\right) \mid t \in\left[a_{2}, b_{2}\right]\right\},
$$

$n=1,2,3, \ldots$, and for each positive integer $k$ the star $S_{k}$ with the center $\left(a_{1}^{k}, a_{2}^{\infty}\right)$ and beams

$$
C_{n}^{k}=\left\{\left(a_{1}^{k}, a_{2}^{n}, t^{\infty}\right) \mid t \in\left[a_{2}, b_{2}\right]\right\},
$$

$n=1,2,3, \ldots$
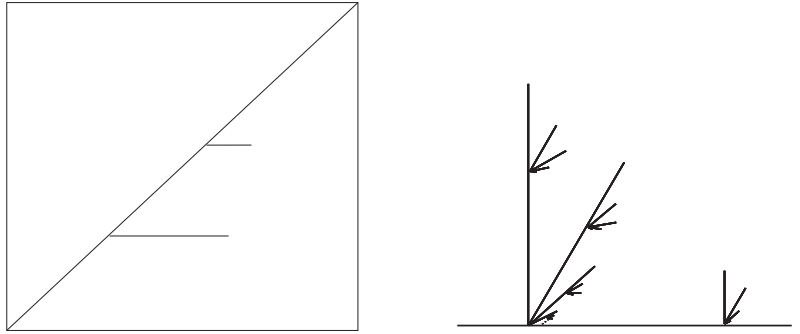

Figure 4. The graph of a 2-comb function and its inverse limit, $b_{1}>a_{2}$

Note that if $b_{1}=a_{2}$ the stars $S_{k}, k=1,2,3, \ldots$, are attached at the end points $\left(a_{1}^{k}, b_{1}^{\infty}\right)$ of $S$, and if $b_{1}>a_{2}$ the stars $S_{k}, k=1,2,3, \ldots$, are attached at the interior points of the maximal free $\operatorname{arcs}\left\{\left(a_{1}^{k}, t^{\infty}\right) \mid t \in\left[a_{1}, b_{1}\right]\right\}$ of $S$, $k=1,2,3, \ldots$

In the following theorem we show that any inverse limit of intervals $[0,1]$ and a single $n$-comb function is a dendrite.

THEOREM 3.5. Let $n$ be any positive integer and let $f:[0,1] \rightarrow 2^{[0,1]}$ be any $n$-comb function. Then $\lim _{\longleftarrow}\{[0,1], f\}_{k=1}^{\infty}$ is a dendrite. 

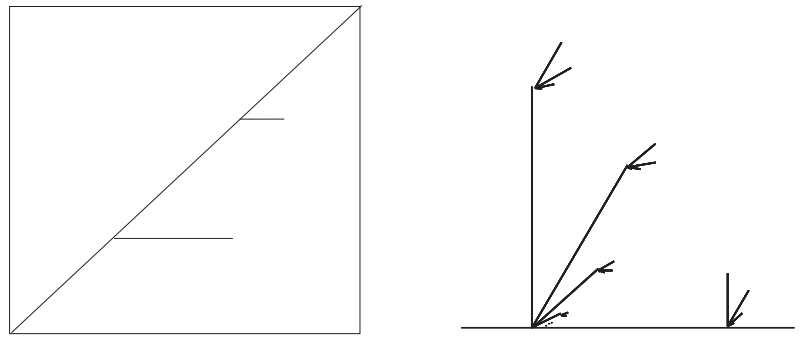

Figure 5 . The graph of a 2 -comb function and its inverse limit, $b_{1}=a_{2}$

Proof. We prove Theorem 3.5 by induction on $n$ by proving the more precise claim that includes also information about maximal free arcs and ramification points in the dendrite. For each positive integer $\ell$, let us introduce the following notation for certain statements that will be used in the inductive proof of the theorem:

(a) $\ell$ The inverse limit $D_{\ell}=\lim _{\longleftarrow}\left\{[0,1], f_{\left(a_{i}, b_{i}\right)_{i=1}^{\ell}}\right\}_{k=1}^{\infty}$ is a dendrite.

(b) $\ell_{\ell}$ The points of the form $\left(x_{1}, x_{2}, x_{3}, \ldots, x_{m}, a_{j}^{\infty}\right) \in D_{\ell}, j \leq \ell$, are exactly the ramification points of $D_{\ell}$.

(c) $\ell$ The points of the form $\left(x_{1}, x_{2}, x_{3}, \ldots, x_{m}, b_{i}^{\infty}\right) \in D_{\ell}, i \leq \ell$, where $m \geq 1, a_{i}=x_{m} \neq b_{i}$, and $b_{i} \notin\left\{a_{i+1}, a_{i+2}, a_{i+3}, \ldots, a_{\ell}\right\}$, are endpoints of $D_{\ell}$.

(d) $)_{\ell}$ All endpoints of $D_{\ell}$ are of such form, except endpoints $\left(0^{\infty}\right)$ and $\left(1^{\infty}\right)$

(e) $)_{\ell}$ The maximal free arc in $D_{\ell}$ having the point

$$
x=\left(x_{1}, x_{2}, x_{3}, \ldots, x_{m}, b_{i}^{\infty}\right)
$$

described in $(\mathrm{c})_{\ell}$ as one endpoint, has

$$
\left(x_{1}, x_{2}, x_{3}, \ldots, x_{m}, a_{\ell}^{\infty}\right)
$$

as the other endpoint if $a_{\ell}<b_{i}$; if $a_{\ell}>b_{i}$ then the maximal free arc in $D_{\ell}$ ending at $x$ equals to the maximal free arc in $D_{\ell-1}$ ending at $x$. (f) $\ell$ The arc with endpoints $\left(a_{\ell}^{\infty}\right)$ and $\left(1^{\infty}\right)$ is a maximal free $\operatorname{arc}$ in $D_{\ell}$.

1. Let $n=1$. There are $a_{1}, b_{1} \in[0,1]$ such that $a_{1}<b_{1}$ and $f=$ $f_{\left(a_{i}, b_{i}\right)_{i=1}^{1}}$. In Example 3.3 it was shown that the inverse limit $D_{1}=$ $\left.\lim _{-\infty}\{0,1], f\right\}_{k=1}^{\infty}$ is a star, and is therefore a dendrite. We see that $\left(a_{1}^{\infty}\right)$ is the only ramification point of $D_{1}$, and that maximal free arcs of $D_{1}$ are exactly the beams $B_{0}=\left\{\left(t^{\infty}\right) \mid t \in\left[0, a_{1}\right]\right\}, B_{0}^{\prime}=\left\{\left(t^{\infty}\right) \mid t \in\right.$ $\left.\left[a_{1}, 1\right]\right\}$ and $B_{k}=\left\{\left(a_{1}^{k}, t^{\infty}\right) \mid t \in\left[a_{1}, b_{1}\right]\right\}, k=1,2,3, \ldots$ of the star $D_{1}$. Note that $(\mathrm{a})_{1}-(\mathrm{f})_{1}$ hold true. 
2. Let $f$ be any $n$-comb function, $n \geq 2$. Without loss of generality we may assume that $f=f_{\left(a_{i}, b_{i}\right)_{i=1}^{n}}$, where $a_{1}<a_{2}<a_{3}<\ldots<a_{n}$.

Let, as the inductive assumption, (a) $)_{n-1}-(\mathrm{f})_{n-1}$ hold true for the function $f_{\left(a_{i}, b_{i}\right)_{i=1}^{n-1} \text {. }}$

We show that the inverse limit

$$
\varliminf_{\longleftarrow}\{[0,1], f\}_{k=1}^{\infty}=D_{n}=\varliminf_{\longleftarrow}\left\{[0,1], f_{\left(a_{i}, b_{i}\right)_{i=1}^{n}}\right\}_{k=1}^{\infty}
$$

satisfies all the above mentioned properties for $\ell=n$.

By the inductive assumption $D_{n-1}=\lim _{\longleftarrow}\left\{[0,1], f_{\left(a_{i}, b_{i}\right)_{i=1}^{n-1}}\right\}_{k=1}^{\infty}$ is a dendrite.

Case 1. $a_{n}>b_{i}$ for each $i=1,2,3, \ldots, n-1$.

In this case any $x \in D_{n} \backslash D_{n-1}$ is of the form $x=\left(a_{n}^{k}, t^{\infty}\right)$, where $k$ is a positive integer and $t \in\left(a_{n}, b_{n}\right]$. Therefore

$$
D_{n}=D_{n-1} \cup S,
$$

where $S=\left\{\left(a_{n}^{k}, t^{\infty}\right) \mid k \in \mathbb{N}, t \in\left[a_{n}, b_{n}\right]\right\}$, and we see that $S$ is a star with the center $\left(a_{n}^{\infty}\right) \in D_{n} \backslash R\left(D_{n-1}\right)$. Obviously (a) $)_{n}$ (f) $)_{n}$ hold true.

Case 2. $a_{n} \leq b_{i}$ for some $i=1,2,3, \ldots, n-1$.

In this case we show that

$$
D_{n}=D_{n-1} \cup(\bigcup \mathcal{S}),
$$

where

(a) $\mathcal{S}=\left\{S_{1}, S_{2}, S_{3}, \ldots\right\}$ is a countable family of stars with centers $c_{1}, c_{2}, c_{3}, \ldots$ respectively, where $c_{1}, c_{2}, c_{3}, \ldots \in D_{n} \backslash R\left(D_{n-1}\right)$, and each of the maximal free $\operatorname{arcs}$ in $D_{n-1}$ contains at most one of these centers,

(b) for each positive integer $i, S_{i} \cap D_{n-1}=\left\{c_{i}\right\}$,

(c) $S_{i} \cap S_{j}=\emptyset$ if $i \neq j$, and

(d) $\lim _{i \rightarrow \infty} \operatorname{diam}\left(S_{i}\right)=0$,

and therefore it will follow that $D_{n}$ is a dendrite by Theorem 2.1, using $(\mathrm{a})_{n-1}$. That will prove $(\mathrm{a})_{n}$.

Any point of $D_{n} \backslash D_{n-1}$ is of the form $\left(x_{1}, x_{2}, x_{3}, \ldots, x_{m}, a_{n}^{k}, t^{\infty}\right)$, where $k$ is a positive integer, $m$ is a nonnegative integer, $t \in\left(a_{n}, b_{n}\right]$, and $x_{m} \neq a_{n}$, and vice versa.

The set

$$
\left\{\left(x_{1}, x_{2}, x_{3}, \ldots, x_{m}, a_{n}^{k}, t^{\infty}\right) \mid k \geq 1, t \in\left[a_{n}, b_{n}\right]\right\}
$$

is a star centered in $\left(x_{1}, x_{2}, x_{3}, \ldots, x_{m}, a_{n}^{\infty}\right)$ having the beams

$$
\left\{\left(x_{1}, x_{2}, x_{3}, \ldots, x_{m}, a_{n}^{k}, t^{\infty}\right) \mid t \in\left[a_{n}, b_{n}\right]\right\},
$$

for each $k \geq 1$. Note that $\mathcal{S}$ is infinite since for each $i$ such that $a_{n} \leq$ $b_{i}$ the family $\mathcal{S}$ contains stars centered at $\left(a_{i}^{k}, a_{n}^{\infty}\right)$ for each positive integer $k$. 
From $f_{\left(a_{i}, b_{i}\right)_{i=1}^{n-1}}^{-1}\left(a_{n}\right)=\left\{a_{n}\right\}$ it follows that if for $x \in D_{n-1}$ and for some positive integer $m, p_{m}(x)=a_{n}$, then $p_{m+1}(x)=a_{n}$. Therefore such $x$ ends with the block $a_{n}^{\infty}$. Let $X_{1}=\left\{\left(a_{n}^{\infty}\right)\right\}$, and let for each positive integer $m \geq 2$,

$$
X_{m}=\left\{x \in D_{n-1} \mid p_{m}(x)=a_{n}, p_{m-1}(x) \neq a_{n}\right\} .
$$

Then $X_{m}$ is a finite set for each $m$. Therefore $X=\bigcup_{m=1}^{\infty} X_{m}$ is a finite or countable infinite subset of $D_{n-1} \backslash R\left(D_{n-1}\right)\left((\mathrm{b})_{n-1}\right.$ is also used). Also, each maximal free arc of $D_{n-1}$ contains at most one $x \in X$. To prove this, we shall for each $x \in X$ find the uniquely determined maximal free arc of $D_{n-1}$ containing $x$. Let

$$
x=\left(x_{1}, x_{2}, x_{3}, \ldots, x_{m}, a_{n}^{\infty}\right) \in X,
$$

where $x_{m} \neq a_{n}$. Then $x_{m}=a_{i}$ for some $i<n$. Note that since $a_{i} \in f_{\left(a_{i}, b_{i}\right)_{i=1}^{n-1}}\left(a_{n}\right)$, it follows that $a_{n} \in\left[a_{i}, b_{i}\right]$ and therefore $b_{i} \geq a_{n}$. Now we distinguish two cases, $b_{i}>a_{n}$ and $b_{i}=a_{n}$.

If $b_{i}>a_{n}$, then $b_{i} \notin\left\{a_{i+1}, a_{i+2}, \ldots, a_{n}\right\}$, hence the point $\left(x_{1}, x_{2}, \ldots, x_{m}, b_{i}^{\infty}\right)$ is an endpoint of $D_{n-1}$ by $(\mathrm{c})_{n-1}$ and the arc

$$
\left\{\left(x_{1}, x_{2}, x_{3}, \ldots, x_{m}, t^{\infty}\right) \mid t \in\left[a_{n-1}, b_{i}\right]\right\}
$$

is a maximal free arc of $D_{n-1}$ by $(\mathrm{e})_{n-1}$. Obviously $x$ belongs to the arc, since $a_{n} \in\left[a_{n-1}, b_{i}\right]$.

If $b_{i}=a_{n}$, then $x$ is an endpoint of $D_{n-1}$ by $(\mathrm{c})_{n-1}$, and clearly it belongs to the maximal free arc $\left\{\left(x_{1}, x_{2}, x_{3}, \ldots, x_{m}, t^{\infty}\right) \mid t \in\right.$ $\left.\left[a_{n-1}, b_{i}\right]\right\}$ of $D_{n-1}$, which is a maximal free $\operatorname{arc}$ in $D_{n-1}$ by $(\mathrm{e})_{n-1}$.

Now, when we have the explicit description of all maximal free arcs in $D_{n-1}$ containing elements of $X$, we see that each such maximal free arc contains exactly one point from $X$.

Take any $x=\left(x_{1}, x_{2}, x_{3}, \ldots, x_{m}, a_{n}^{\infty}\right) \in X$, where $x_{m} \neq a_{n}$. Then $x_{m}=a_{i}$ for some $i<n$. For each positive integer $k$, let

$$
B_{k}=\left\{\left(x_{1}, x_{2}, x_{3}, \ldots, x_{m}, a_{n}^{k}, t^{\infty}\right) \mid t \in\left[a_{n}, b_{n}\right]\right\} .
$$

Obviously, $B_{k}$ is an arc in $D_{n}$ and $S(x)=\bigcup_{k=1}^{\infty} B_{k}$ is a star centered at $x$. The diameter of $S(x)$ satisfies

$$
\operatorname{diam}(S(x)) \leq D\left(\left(x_{1}, x_{2}, \ldots, x_{m}, 0^{\infty}\right),\left(x_{1}, x_{2}, \ldots, x_{m}, 1^{\infty}\right)\right) \leq \frac{1}{2^{m+1}} .
$$

Since for each $m$ there are only finitely many such points $x \in X\left(X_{m}\right.$ is finite), it follows that the set $\mathcal{S}=\{S(x) \mid x \in X\}$ is finite or it can be presented as $\mathcal{S}=\left\{S_{1}, S_{2}, S_{3}, \ldots\right\}$. From the above upper bound for the diameters of the stars in the infinite case it follows that $\lim _{i \rightarrow \infty} \operatorname{diam}\left(S_{i}\right)=0$.

Take any point $x \in D_{n} \backslash D_{n-1}$. As already noticed, it is of the form $x=\left(x_{1}, x_{2}, x_{3}, \ldots, x_{m}, a_{n}^{k}, t^{\infty}\right)$, where $k$ is a positive integer, $m$ is 
a nonnegative integer, $t \in\left(a_{n}, b_{n}\right]$, and $x_{m} \neq a_{n}$. Therefore $x \in S(y)$, where $y=\left(x_{1}, x_{2}, \ldots, x_{m}, a_{n}^{\infty}\right) \in X$. Therefore

$$
D_{n} \backslash D_{n-1}=\left(\bigcup_{x \in X} S(x)\right) \backslash X=(\bigcup \mathcal{S}) \backslash X,
$$

and finally

$$
D_{n}=D_{n-1} \cup\left(\bigcup_{x \in X} S(x)\right)=D_{n-1} \cup(\bigcup \mathcal{S}),
$$

proving $(\mathrm{a})_{n}$.

To prove that the points of the form $x=\left(x_{1}, x_{2}, x_{3}, \ldots, x_{m}, b_{i}^{\infty}\right) \in$ $D_{n}$, where $i \leq n, x_{m}=a_{i}$, and $b_{i} \notin\left\{a_{i+1}, a_{i+2}, \ldots, a_{n}\right\}$, are endpoints of $D_{n}$, we distinguish two cases. If $i \leq n-1$ then $x \in D_{n-1}$, and then $x$ is an endpoint of $D_{n-1}$ by (c) $)_{n-1}$, since $b_{i} \notin\left\{a_{i+1}, a_{i+2}, a_{i+3}, \ldots, a_{n-1}\right\}$. Since $b_{i} \neq a_{n}$ the only star attached to the maximal free arc in $D_{n-1}$ ending at $x$ is centered at a point that differs from $x$, or no star is attached to that arc at all, it follows that $x \in E\left(D_{n}\right)$. If $i=n$, then $b_{i}=b_{n}$, and therefore $x$ is an endpoint of a star from $\mathcal{S}$. That proves $(\mathrm{c})_{n}$.

Also each endpoint of $D_{n}$ which belongs to $D_{n-1}$, is also an endpoint in $D_{n-1}$, therefore it is of the form

$$
x=\left(x_{1}, x_{2}, x_{3}, \ldots, x_{m}, b_{i}^{\infty}\right) \in D_{n},
$$

where $x_{m} \neq b_{i}$ and $b_{i} \notin\left\{a_{i+1}, a_{i+2}, a_{i+3}, \ldots, a_{n-1}\right\}$, by $(\mathrm{d})_{n-1}$. Points of such form with $b_{i}=a_{n}$ are centers of the newly attached stars and therefore are not endpoints of $D_{n}$. It follows that $b_{i} \neq a_{n}$, and therefore $b_{i} \notin\left\{a_{i+1}, a_{i+2}, a_{i+3}, \ldots, a_{n}\right\}$. Each endpoint of $D_{n}$, which belongs to $D_{n} \backslash D_{n-1}$, is necessarily an endpoint of a newly attached star and therefore is of the form $x=\left(x_{1}, x_{2}, x_{3}, \ldots, x_{m}, b_{n}^{\infty}\right)$, $a_{n}=x_{m} \neq b_{n}$. Additional condition $b_{i} \notin\left\{a_{i+1}, a_{i+2}, a_{i+3}, \ldots, a_{n}\right\}$ is satisfied vacuously for $i=n$. Obviously $\left(0^{\infty}\right)$ and $\left(1^{\infty}\right)$ are endpoints of $D_{n}$, too. That proves $(\mathrm{d})_{n}$.

Let $x=\left(x_{1}, x_{2}, x_{3}, \ldots, x_{m}, b_{i}^{\infty}\right) \in D_{n}$ be any endpoint of $D_{n}$ mentioned in (c) $)_{n}$, where $a_{i}=x_{m} \neq b_{i}$ and $b_{i} \notin\left\{a_{i+1}, a_{i+2}, a_{i+3}, \ldots, a_{n}\right\}$. If $i<n$ then by $(\mathrm{c})_{n-1} x$ is an endpoint of $D_{n-1}$. If $a_{n}<b_{i}$ then we have already proved that a new star centered at $\left(x_{1}, x_{2}, x_{3}, \ldots, x_{m}, a_{n}^{\infty}\right)$ is attached to the maximal free arc of $D_{n-1}$ ending at $x$, and since no other star was attached to this arc it follows that the point $\left(x_{1}, x_{2}, x_{3}, \ldots, x_{m}, a_{n}^{\infty}\right)$ is the other endpoint of the maximal free arc of $D_{n}$ ending at $x$. If $a_{n}>b_{i}$ then no star was attached to the maximal free arc of $D_{n-1}$ ending at $x=\left(x_{1}, x_{2}, x_{3}, \ldots, x_{m}, b_{i}^{\infty}\right)$, and therefore it remained a maximal free arc of $D_{n}$ as well. This proves (e) $)_{n}$. 
By (f) $)_{n-1}$ the maximal free arc of $D_{n-1}$ having $\left(1^{\infty}\right)$ as one endpoint has $\left(a_{n-1}^{\infty}\right)$ as the other endpoint. Since a star centered at $\left(a_{n}^{\infty}\right)$ was attached to $D_{n-1}$, and since no other star was attached to the above mentioned arc, (f) $)_{n}$ follows.

Finally $(\mathrm{b})_{n}$ follows from (b) ${ }_{n-1}$ and from the fact that at each point of the form $\left(x_{1}, x_{2}, x_{3}, \ldots, x_{m}, a_{n}^{\infty}\right) \in D_{n}$ a new star was attached to $D_{n-1}$, as shown above.

In the following remark we extract certain parts of the above proof for later use.

REMARK 3.6. Let $n$ be a positive integer.

1. For each positive integer $n$ and for each $y \in D_{n}, y$ is either of the form $y=\left(t^{\infty}\right), t \in[0,1]$, or of the form $y=\left(a_{i_{1}}^{k_{1}}, a_{i_{2}}^{k_{2}}, a_{i_{3}}^{k_{3}}, \ldots, a_{i_{m}}^{k_{m}}, t^{\infty}\right)$, where $m$ is a positive integer and for each $\ell \leq m$ it holds that $i_{\ell} \leq n$, $k_{\ell}>0, a_{i_{\ell}}<a_{i_{\ell+1}} \leq b_{i_{\ell}}$, and $a_{i_{m}} \leq t \leq b_{i_{m}}$.

2. Any point of $D_{n+1} \backslash D_{n}$ is of the form

$$
\left(x_{1}, x_{2}, x_{3}, \ldots, x_{m}, a_{n+1}^{k}, t^{\infty}\right),
$$

where $k$ is a positive integer, $m$ is a nonnegative integer, $t \in$ $\left(a_{n+1}, b_{n+1}\right]$, and $x_{m} \neq a_{n+1}$.

3. $x \in D_{n}$ is a ramification point in $D_{n}$ if and only if there are positive integers $m$ and $j \leq n$, such that $p_{k}(x)=a_{j}$ for each positive integer $k \geq m$.

Definition 3.7. We will use $D_{n}$ to denote the dendrite

$$
D_{n}=\lim _{\longleftarrow}\left\{[0,1], f_{\left(a_{i}, b_{i}\right)_{i=1}^{n}}\right\}_{k=1}^{\infty} .
$$

Next we define functions that we shall use later in proof of the main result.

Definition 3.8. We define the function $f_{n}: D_{n+1} \rightarrow D_{n}$ by

$$
f_{n}(x)=\left\{\begin{array}{l}
g_{n}(x) \quad ; \quad x \in \mathrm{Cl}\left(D_{n+1} \backslash D_{n}\right), \\
x \quad ; \quad x \in D_{n},
\end{array}\right.
$$

where $g_{n}: \mathrm{Cl}\left(D_{n+1} \backslash D_{n}\right) \rightarrow D_{n}$ is defined as follows. Any point of $\mathrm{Cl}\left(D_{n+1} \backslash\right.$ $\left.D_{n}\right)$ is of the form

$$
x=\left(x_{1}, x_{2}, x_{3}, \ldots, x_{m}, a_{n+1}^{k}, t^{\infty}\right),
$$

where $k$ is a positive integer, $m$ is a nonnegative integer, $t \in\left[a_{n+1}, b_{n+1}\right]$, and $x_{m} \neq a_{n+1}$ (see Remark 3.6), and we define

$$
g_{n}(x)=\left(x_{1}, x_{2}, x_{3}, \ldots, x_{m}, a_{n+1}^{\infty}\right) .
$$

Note that $f_{n}$ is continuous for each $n$ by [19, Theorem 7.3, p. 108]. 
Lemma 3.9. Let $x \in D_{n}$.

1. If

$$
x=\left(a_{i_{1}}^{k_{1}}, a_{i_{2}}^{k_{2}}, a_{i_{3}}^{k_{3}}, \ldots, a_{i_{j}}^{k_{j}}, t^{\infty}\right) \in D_{n},
$$

where $j>0, i_{1}, i_{2}, i_{3}, \ldots, i_{j} \leq n, a_{i_{1}}<a_{i_{2}}<\cdots<a_{i_{j}}, k_{1}, k_{2}, \ldots, k_{j}>$ 0 , and $t \in\left[a_{i_{j}}, b_{i_{j}}\right]$, then for each

$$
y \in f_{n}^{-1}(x)
$$

and for each $i \leq k_{1}+k_{2}+k_{3}+\ldots+k_{j}+1$ it holds that $p_{i}(x)=p_{i}(y)$.

2. If $x=\left(t^{\infty}\right), t \in[0,1]$, then for each

$$
y \in f_{n}^{-1}(x)
$$

it holds that $p_{1}(x)=p_{1}(y)=t$.

Proof. If $y \in D_{n}$, then $y=x$ and the claim is obviously true. Note that in Case 1. from $t=a_{i_{j}}$ it follows that $y \in D_{n}$.

If $y \in D_{n+1} \backslash D_{n}$, then by 2 . from Remark $3.6 y$ is of the form

$$
\left(x_{1}, x_{2}, x_{3}, \ldots, x_{m}, a_{n+1}^{k}, s^{\infty}\right),
$$

where $k$ is a positive integer, $m$ is a nonnegative integer, $s \in\left(a_{n+1}, b_{n+1}\right]$, and $x_{m} \neq a_{n+1}$. Then $x=f_{n}(y)=g_{n}(y)=\left(x_{1}, x_{2}, x_{3}, \ldots, x_{m}, a_{n+1}^{\infty}\right)$.

In Case 1. in the remaining case $t \neq a_{i_{j}}$ it follows that $m=k_{1}+$ $k_{2}+k_{3}+\ldots+k_{j}$ and $t=a_{n+1}$. Therefore $\left(x_{1}, x_{2}, x_{3}, \ldots, x_{m}, a_{n+1}\right)=$ $\left(a_{i_{1}}^{k_{1}}, a_{i_{2}}^{k_{2}}, a_{i_{3}}^{k_{3}}, \ldots, a_{i_{j}}^{k_{j}}, t\right)$.

In Case 2. it follows that $m=0$ and $t=a_{n+1}$.

Lemma 3.10. Let $x=\left(x_{1}, x_{2}, x_{3}, \ldots, x_{m}, a_{n+1}^{\infty}\right) \in D_{n}$, where $n$ is a positive integer, $m$ is a nonnegative integer, and $x_{m} \neq a_{n+1}$. Then $f_{n}^{-1}(x)$ is a star centered in $x$.

Proof. From what we have seen in the proof of Lemma 3.9 it follows that

$$
f_{n}^{-1}(x)=\bigcup_{k=1}^{\infty}\left\{\left(x_{1}, x_{2}, x_{3}, \ldots, x_{m}, a_{n+1}^{k}, s^{\infty}\right) \mid s \in\left[a_{n+1}, b_{n+1}\right]\right\},
$$

and for each $k$ the set

$$
B_{k}=\left\{\left(x_{1}, x_{2}, x_{3}, \ldots, x_{m}, a_{n+1}^{k}, s^{\infty}\right) \mid s \in\left[a_{n+1}, b_{n+1}\right]\right\}
$$

is an arc with endpoints $x$ and $\left(x_{1}, x_{2}, x_{3}, \ldots, x_{m}, a_{n+1}^{k}, b_{n+1}^{\infty}\right), B_{i} \cap B_{j}=\{x\}$ for any $i \neq j$, and $\lim _{k \rightarrow \infty} \operatorname{diam}\left(B_{k}\right)=0$.

Let $\left\{\left(a_{n}, b_{n}\right)\right\}_{n=1}^{\infty}$ be any sequence in $[0,1] \times[0,1]$, such that $a_{n}<b_{n}$ for each positive integer $n$, and $a_{i} \neq a_{j}$ whenever $i \neq j$. Next denote by $A\left(a_{n}, b_{n}\right)_{n=1}^{\infty}$ the union

$$
A\left(a_{n}, b_{n}\right)_{n=1}^{\infty}=\bigcup_{n=1}^{\infty}\left(\left[a_{n}, b_{n}\right] \times\left\{a_{n}\right\}\right) \subseteq[0,1] \times[0,1] .
$$


and by $G\left(a_{n}, b_{n}\right)_{n=1}^{\infty}$ the subset of $[0,1] \times[0,1]$, defined by

$$
G\left(a_{n}, b_{n}\right)_{n=1}^{\infty}=A \cup A\left(a_{n}, b_{n}\right)_{n=1}^{\infty},
$$

where $A=\{(t, t) \mid t \in[0,1]\}$ as above.

It is easy to see that $\pi_{1}\left(G\left(a_{i}, b_{i}\right)_{i=1}^{n}\right)=\pi_{2}\left(G\left(a_{i}, b_{i}\right)_{i=1}^{n}\right)=[0,1]$.

Obviously $G\left(a_{n}, b_{n}\right)_{n=1}^{\infty}$ is not necessarily closed in $[0,1] \times[0,1]$. The following theorem gives a whole family of sets $G\left(a_{n}, b_{n}\right)_{n=1}^{\infty}$ that are closed in $[0,1] \times[0,1]$

THEOREM 3.11. Let $\left\{\left(a_{n}, b_{n}\right)\right\}_{n=1}^{\infty}$ be any sequence in $[0,1] \times[0,1]$, such that

1. $a_{n}<b_{n}$ for each positive integer $n$,

2. $a_{i} \neq a_{j}$ whenever $i \neq j$,

3. $\lim _{n \rightarrow \infty}\left(b_{n}-a_{n}\right)=0$.

Then $G\left(a_{n}, b_{n}\right)_{n=1}^{\infty}$ is a closed subset of $[0,1] \times[0,1]$.

Proof. Let $\left\{x_{n}\right\}_{n=1}^{\infty}$ be any sequence in $G\left(a_{n}, b_{n}\right)_{n=1}^{\infty}$, which is convergent in $[0,1] \times[0,1]$ with the limit $x_{0} \in[0,1] \times[0,1]$. We show that $x_{0} \in$ $G\left(a_{n}, b_{n}\right)_{n=1}^{\infty}$.

If there are positive integers $k$ and $n_{0}$, such that $x_{n} \in\left[a_{k}, b_{k}\right] \times\left\{a_{k}\right\}$ for each $n \geq n_{0}$, then, since $\left[a_{k}, b_{k}\right] \times\left\{a_{k}\right\}$ is compact, $x_{0} \in\left[a_{k}, b_{k}\right] \times\left\{a_{k}\right\}$ and therefore $x_{0} \in G\left(a_{n}, b_{n}\right)_{n=1}^{\infty}$. Otherwise there are strictly increasing sequences $\left\{i_{n}\right\}_{n=1}^{\infty}$ and $\left\{j_{n}\right\}_{n=1}^{\infty}$ of integers, such that $x_{i_{n}} \in\left(\left[a_{j_{n}}, b_{j_{n}}\right] \times\left\{a_{j_{n}}\right\}\right) \cup A$, where $A=\{(t, t) \in[0,1] \times[0,1] \mid t \in[0,1]\}$, for each positive integer $n$. Since $\lim _{n \rightarrow \infty}\left(b_{n}-a_{n}\right)=0$, it follows that $x_{0} \in A$ and therefore $x_{0} \in G\left(a_{n}, b_{n}\right)_{n=1}^{\infty}$.

Therefore by Theorem 2.3 it follows that for any sequence $\left\{\left(a_{n}, b_{n}\right)\right\}_{n=1}^{\infty}$ satisfying

1. $a_{n}<b_{n}$ for each positive integer $n$,

2. $a_{i} \neq a_{j}$ whenever $i \neq j$,

3. $\lim _{n \rightarrow \infty}\left(b_{n}-a_{n}\right)=0$,

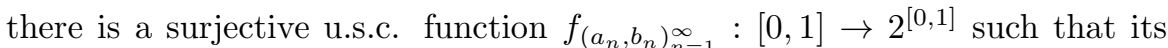
graph $\Gamma\left(f_{\left(a_{n}, b_{n}\right)_{n=1}^{\infty}}\right)$ equals to $G\left(a_{n}, b_{n}\right)_{n=1}^{\infty}$, since $G\left(a_{n}, b_{n}\right)_{n=1}^{\infty}$ is a closed subset of $[0,1] \times[0,1]$ by Theorem 3.11 , and since

$$
\pi_{1}\left(G\left(a_{i}, b_{i}\right)_{i=1}^{n}\right)=\pi_{2}\left(G\left(a_{i}, b_{i}\right)_{i=1}^{n}\right)=[0,1] .
$$

Definition 3.12. Let $\left\{\left(a_{n}, b_{n}\right)\right\}_{n=1}^{\infty}$ be any sequence in $[0,1] \times[0,1]$, such that

1. $a_{n}<b_{n}$ for each positive integer $n$,

2. $a_{i} \neq a_{j}$ whenever $i \neq j$,

3. $\lim _{n \rightarrow \infty}\left(b_{n}-a_{n}\right)=0$. 
Then $f_{\left(a_{n}, b_{n}\right)_{n=1}^{\infty}}$ is called the comb function with respect to $\left\{\left(a_{n}, b_{n}\right)\right\}_{n=1}^{\infty}$.

We also say that $f:[0,1] \rightarrow 2^{[0,1]}$ is a comb function, if $f$ is the comb function with respect to some sequence $\left\{\left(a_{n}, b_{n}\right)\right\}_{n=1}^{\infty}$ in $[0,1] \times[0,1]$ satisfying 1., 2. and 3.

THEOREM 3.13. Let $f:[0,1] \rightarrow 2^{[0,1]}$ be the comb function with respect to the sequence $\left\{\left(a_{n}, b_{n}\right)\right\}_{n=1}^{\infty}$. Then

$$
\varliminf_{\longleftarrow}\{[0,1], f\}_{k=1}^{\infty}=\mathrm{Cl}\left(\bigcup_{n=1}^{\infty} D_{n}\right) .
$$

Proof. Obviously, since $\varliminf_{\longleftarrow}\{[0,1], f\}_{k=1}^{\infty}$ is closed in $\prod_{n=1}^{\infty}[0,1]$,

$$
\lim _{\longleftarrow}\{[0,1], f\}_{k=1}^{\infty} \supseteq \mathrm{Cl}\left(\bigcup_{n=1}^{\infty} D_{n}\right) \text {. }
$$

Next we show that

$$
\varliminf_{\longleftarrow}\{[0,1], f\}_{k=1}^{\infty} \subseteq \mathrm{Cl}\left(\bigcup_{n=1}^{\infty} D_{n}\right) .
$$

Let $x \in \lim _{\longleftarrow}\{[0,1], f\}_{k=1}^{\infty} \backslash \bigcup_{n=1}^{\infty} D_{n}$. Obviously $x$ is of the form

$$
x=\left(a_{i_{1}}, a_{i_{2}}, a_{i_{3}}, \ldots\right),
$$

where $\left\{a_{i_{n}} \mid n=1,2,3, \ldots\right\}$ is an infinite subset of $\left\{a_{n} \mid n=1,2,3, \ldots\right\}$.

Take any open ball $U=B(x, \varepsilon)$ in $\prod_{n=1}^{\infty}[0,1]$ with respect to the metric $D$. Let $m$ be a positive integer such that $\frac{1}{2^{m}}<\varepsilon$. Then

$$
\left(a_{i_{1}}, a_{i_{2}}, \ldots, a_{i_{m-1}}, a_{i_{m}}^{\infty}\right) \in U \cap D_{i_{m}} .
$$

In the above proof we noticed that any $x \in \varliminf_{n}\{[0,1], f\}_{k=1}^{\infty} \backslash \bigcup_{n=1}^{\infty} D_{n}$ is of the form $x=\left(a_{i_{1}}, a_{i_{2}}, a_{i_{3}}, \ldots\right)$, where $\left\{a_{i_{n}} \mid n=1,2,3, \ldots\right\}$ is an infinite subset of $\left\{a_{n} \mid n=1,2,3, \ldots\right\}$. We can make this statement more precise taking into account the definitions of inverse limits and comb functions as follows:

Remark 3.14. Let $f:[0,1] \rightarrow 2^{[0,1]}$ be the comb function with respect to the sequence $\left\{\left(a_{n}, b_{n}\right)\right\}_{n=1}^{\infty}$. Any point $x \in \lim _{\longleftarrow}\{[0,1], f\}_{k=1}^{\infty} \backslash \bigcup_{n=1}^{\infty} D_{n}$ is of the form

$$
\left(a_{i_{1}}^{k_{1}}, a_{i_{2}}^{k_{2}}, a_{i_{3}}^{k_{3}}, \ldots\right)
$$

where for each $\ell$ it holds that $k_{\ell}>0$ and $a_{i_{\ell}}<a_{i_{\ell+1}} \leq b_{i_{\ell}}$.

In Examples 3.15 and 3.16 we show that there are comb functions $f$, such that the inverse limits $\varliminf_{\longleftarrow}\{[0,1], f\}_{k=1}^{\infty}$ are not dendrites. 


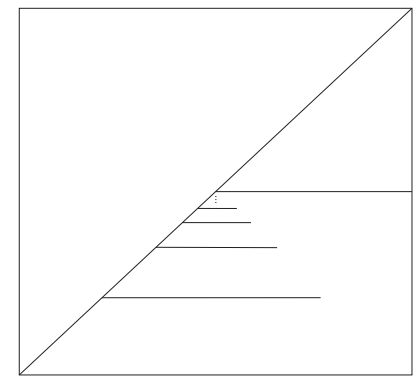

Figure 6 . The graph of the comb function from Example 3.15

EXAMPLE 3.15. Let $\left(a_{1}, b_{1}\right)=\left(\frac{1}{2}, 1\right)$, and let for each positive integer $n \geq 2,\left(a_{n}, b_{n}\right)=\left(\frac{1}{2}-\frac{1}{n+1}, \frac{1}{2}+\frac{1}{n+1}\right)$. We show that $\varliminf_{\longleftarrow}\left\{[0,1], f_{\left(a_{n}, b_{n}\right)_{n=1}^{\infty}}\right\}_{k=1}^{\infty}$ is not locally connected, and therefore it is not a dendrite. Let

$$
x_{0}=\left(\frac{1}{2}, \frac{1}{2}, 1^{\infty}\right) \in \lim _{\longleftarrow}\left\{[0,1], f_{\left(a_{n}, b_{n}\right)_{n=1}^{\infty}}\right\}_{k=1}^{\infty}
$$

and $\varepsilon=\min \left\{d\left(x_{0}, K\right), \frac{1}{2^{3} \cdot 6}\right\}>0$, where $K=\left\{\left(t^{\infty}\right) \mid t \in[0,1]\right\}$. Let $r \leq \varepsilon$

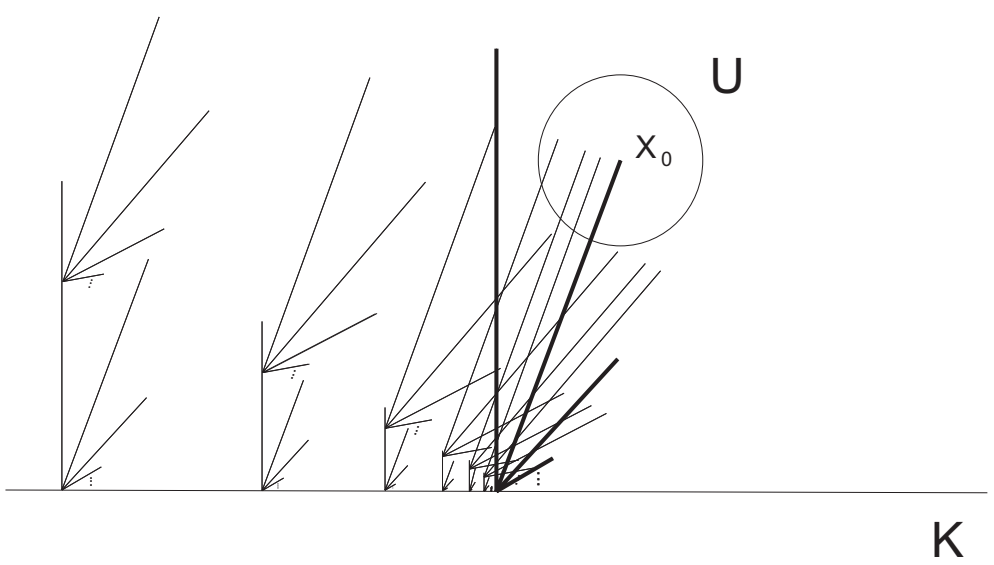

Figure 7. The continuum from Example 3.15

and $y=\left(y_{1}, y_{2}, y_{3}, \ldots\right) \in B\left(x_{0}, r\right) \cap \varliminf_{\lfloor}\left\{[0,1], f_{\left(a_{n}, b_{n}\right)_{n=1}^{\infty}}\right\}_{k=1}^{\infty}$ be arbitrarily chosen. Then, since $r>D\left(x_{0}, y\right) \geq \frac{1-y_{3}}{2^{3}}$, it follows that $y_{3}>1-2^{3} r$. Therefore $y_{3}>1-2^{3} r \geq 1-\frac{2^{3}}{6 \cdot 2^{3}}=\frac{5}{6}$, and hence $y_{i}=y_{3}$ for each $i \geq 3$. Furthermore, $y_{2} \in f\left(y_{3}\right)=\left\{\frac{1}{2}, y_{3}\right\}$. If $y_{2}=y_{3}$, then

$$
D\left(x_{0}, y\right) \geq \frac{y_{2}-\frac{1}{2}}{2^{2}}>\frac{\frac{5}{6}-\frac{1}{2}}{2^{2}}=\frac{1}{12}>r,
$$


a contradiction. Therefore $y_{2}=\frac{1}{2}$, and hence $y_{1} \in f\left(\frac{1}{2}\right)$. Clearly there is a positive integer $n$, such that $y_{1}=a_{n}$ and $\frac{\frac{1}{2}-a_{n}}{2}=\frac{x_{1}-y_{1}}{2}<r$.

Therefore for each $r \leq \varepsilon, y \in B\left(x_{0}, r\right)$ if and only if there is a positive integer $n$, such that $y=\left(a_{n}, \frac{1}{2}, t^{\infty}\right)$, where $\frac{\frac{1}{2}-a_{n}}{2}<r$ and $t>1-2^{3} r$.

Therefore for each $r \leq \varepsilon$ the intersection $B\left(x_{0}, r\right) \cap \lim _{\longleftarrow}\left\{[0,1], f_{\left(a_{n}, b_{n}\right)_{n=1}^{\infty}}\right\}_{k=1}^{\infty}$ is the union of countably many mutually disjoint intervals

$$
\left\{\left(a_{n}, \frac{1}{2}, t^{\infty}\right) \mid t \in\left(1-2^{3} r, 1\right]\right\},
$$

where $\frac{\frac{1}{2}-a_{n}}{2}<r$. See Fig. 7 .

EXAMPLE 3.16. Let $\left(a_{1}, b_{1}\right)=\left(\frac{1}{2}, 1\right)$, and let for each positive integer $n \geq 2,\left(a_{n}, b_{n}\right)=\left(\frac{1}{2}-\frac{1}{n}, \frac{1}{2}\right)$. A similar argument as in Example 3.15 shows

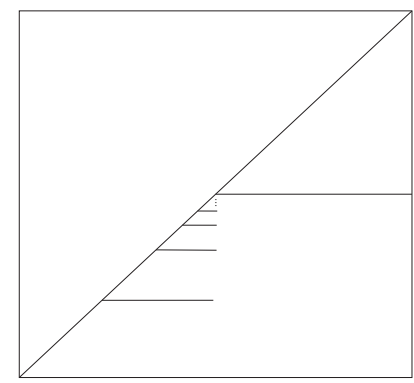

Figure 8. The graph of the comb function in Example 3.16

that the inverse limit $\lim _{\longleftarrow}\left\{[0,1], f_{\left(a_{n}, b_{n}\right)_{n=1}^{\infty}}^{\infty}\right\}_{k=1}^{\infty}$ is not locally connected, and therefore it is not a dendrite.

In Theorem 3.20 we prove that under rather weak additional assumptions the inverse limit of a comb function is a dendrite. Essentially, the conditions say that the only comb functions for which the inverse limits are not dendrites are similar to those from Examples 3.15 and 3.16. Before stating and proving the theorem we introduce the necessary notion of admissible sequences and prove a few lemmas.

DeFinition 3.17. The sequence $\left\{\left(a_{n}, b_{n}\right)\right\}_{n=1}^{\infty}$ in $[0,1] \times[0,1]$ is admissible if for each positive integer $n$ there is a positive integer $\mu(n) \geq n$, such that for each $m \geq \mu(n)$ it holds that if $a_{m}<a_{n}$, then $b_{m}<a_{n}$.

Lemma 3.18. Let $f:[0,1] \rightarrow 2^{[0,1]}$ be any comb function with respect to a sequence $\left\{\left(a_{n}, b_{n}\right)\right\}_{n=1}^{\infty}$, and let

$$
x=\left(a_{i_{1}}^{k_{1}}, a_{i_{2}}^{k_{2}}, a_{i_{3}}^{k_{3}}, \ldots, a_{i_{j}}^{k_{j}}, t^{\infty}\right) \in D_{n},
$$


$j \geq 0, i_{1}, i_{2}, i_{3}, \ldots, i_{j} \leq n, a_{i_{1}}<a_{i_{2}}<\cdots<a_{i_{j}}, k_{1}, k_{2}, \ldots, k_{j}>0$, and $t \in\left[a_{i_{j}}, b_{i_{j}}\right]$. Let $f_{\ell}$ be the functions defined in Definition 3.8. Then for each

$$
y \in \mathrm{Cl}\left(\bigcup_{k \geq n}\left(f_{n} \circ f_{n+1} \circ \ldots \circ f_{k}\right)^{-1}(x)\right)
$$

and for each $i \leq k_{1}+k_{2}+k_{3}+\ldots+k_{j}+1$ it holds that $p_{i}(x)=p_{i}(y)$ (where $x$ and $y$ are interpreted as elements of $\left.\Pi_{n=1}^{\infty}[0,1]\right)$.

Proof. By induction on $k-n$ we prove the following claim:

for each

$$
y \in\left(f_{n} \circ f_{n+1} \circ \ldots \circ f_{k}\right)^{-1}(x)
$$

and for each $i \leq k_{1}+k_{2}+k_{3}+\ldots+k_{j}+1$ it holds that $p_{i}(x)=p_{i}(y)$.

For $k-n=0$ the statement holds true by Lemma 3.9 (part 1 . for $j>0$ and part 2. for $j=0$ ).

Let $k-n=\ell$ and assume that the claim is true for $\ell-1$. Since

$$
\left(f_{n} \circ f_{n+1} \circ \ldots \circ f_{k}\right)^{-1}(x)=\bigcup_{z \in\left(f_{n} \circ f_{n+1} \circ \ldots \circ f_{k-1}\right)^{-1}(x)} f_{k}^{-1}(z)
$$

for any $y \in\left(f_{n} \circ f_{n+1} \circ \ldots \circ f_{k}\right)^{-1}(x)$ we choose $z \in\left(f_{n} \circ f_{n+1} \circ \ldots \circ f_{k-1}\right)^{-1}(x)$ such that $y \in f_{k}^{-1}(z)$. By the induction assumption $p_{i}(x)=p_{i}(z)$ for each $i \leq k_{1}+k_{2}+k_{3}+\ldots+k_{j}+1$, and by Lemma $3.9 p_{i}(y)=p_{i}(z)$ again for each $i \leq k_{1}+k_{2}+k_{3}+\ldots+k_{j}+1$. This completes the proof since the limits of sequences of points with the required property have the property.

We will also need the following lemma about point preimages.

Lemma 3.19. Let $f:[0,1] \rightarrow 2^{[0,1]}$ be the comb function with respect to any admissible sequence $\left\{\left(a_{n}, b_{n}\right)\right\}_{n=1}^{\infty}$. For each $\varepsilon>0$ there is a positive integer $k$ such that

$$
\operatorname{diam}\left(\bigcup_{n \geq k}\left(f_{k} \circ f_{k+1} \circ \ldots \circ f_{n}\right)^{-1}(p)\right)<\varepsilon
$$

for each $p \in D_{k}$, where maps $f_{n}$ are defined as in Definition 3.8.

Proof. Let $\varepsilon>0$ and $m$ be a positive integer such that $\frac{1}{2^{m-1}}<\varepsilon$. Also let $n_{0}>m$ be any positive integer such that for each $n \geq n_{0}$, it holds that $b_{n}-a_{n}<\frac{\varepsilon}{m}$. For each positive integer $\ell$, let $\mu(\ell)$ be a positive integer such that for each $n \geq \mu(\ell)$ it holds that if $a_{n}<a_{\ell}$, then $b_{n}<a_{\ell}$ (here we use the admissibility of the sequence $\left.\left\{\left(a_{n}, b_{n}\right)\right\}_{n=1}^{\infty}\right)$. 
Let

$$
\begin{aligned}
k_{0} & =\max \left\{n_{0}, \mu(1), \mu(2), \mu(3), \ldots, \mu\left(n_{0}\right)\right\}, \\
k_{1} & =\max \left\{n_{0}, \mu(1), \mu(2), \mu(3), \ldots, \mu\left(k_{0}\right)\right\}, \\
k_{2} & =\max \left\{n_{0}, \mu(1), \mu(2), \mu(3), \ldots, \mu\left(k_{1}\right)\right\}, \\
& \vdots \\
k_{m} & =\max \left\{n_{0}, \mu(1), \mu(2), \mu(3), \ldots, \mu\left(k_{m-1}\right)\right\} .
\end{aligned}
$$

Then we show that

$$
k=\max \left\{n_{0}, \mu(1), \mu(2), \mu(3), \ldots, \mu\left(k_{m}\right)\right\}
$$

is a positive integer, such that

$$
\operatorname{diam}\left(\bigcup_{n \geq k}\left(f_{k} \circ f_{k+1} \circ \ldots \circ f_{n}\right)^{-1}(p)\right)<\varepsilon
$$

for each $p \in D_{k}$.

Take any $p \in D_{k}$. Then by 1 . from Remark $3.6 p$ is either of the form $p=\left(t^{\infty}\right), t \in[0,1]$, or of the form $p=\left(p_{1}, p_{2}, p_{3}, \ldots, p_{j}, t^{\infty}\right)$, where $p_{j}=a_{s}$ for some $s \leq k$ and $t \in\left(a_{s}, b_{s}\right]$.

Clearly, it holds that

$$
\operatorname{diam}\left(\bigcup_{n \geq k}\left(f_{k} \circ f_{k+1} \circ \ldots \circ f_{n}\right)^{-1}(p)\right) \leq \operatorname{diam}\left(\bigcup_{n \geq k}\left(f_{k} \circ f_{k+1} \circ \ldots \circ f_{n}\right)^{-1}\left(t^{\infty}\right)\right)
$$

since

$$
\begin{aligned}
& \left(f_{k} \circ f_{k+1} \circ \ldots \circ f_{n}\right)^{-1}\left(\left(p_{1}, p_{2}, p_{3}, \ldots, p_{j}, t^{\infty}\right)\right) \\
& \quad=\left\{\left(p_{1}, p_{2}, p_{3}, \ldots, p_{j}, x_{1}, x_{2}, x_{3}, \ldots\right) \mid\left(x_{1}, x_{2}, x_{3}, \ldots\right)\right. \\
& \left.\quad \in\left(f_{k} \circ f_{k+1} \circ \ldots \circ f_{n}\right)^{-1}\left(t^{\infty}\right)\right\} .
\end{aligned}
$$

If $t \neq a_{i}$ for all $i>k$, then $\bigcup_{n \geq k}\left(f_{k} \circ f_{k+1} \circ \ldots f_{n}\right)^{-1}\left(t^{\infty}\right)=\left\{\left(t^{\infty}\right)\right\}$, and therefore $\operatorname{diam}\left(\bigcup_{n \geq k}\left(f_{k} \circ f_{k+1} \circ \ldots f_{n}\right)^{-1}\left(t^{\infty}\right)\right)=0$.

If $t=a_{i}$ for some $i>k$, then we shall prove that

$$
\operatorname{diam}\left(\bigcup_{n \geq k}\left(f_{k} \circ f_{k+1} \circ \ldots \circ f_{n}\right)^{-1}\left(t^{\infty}\right)\right)<\varepsilon
$$

by proving that

$$
D\left(y,\left(t^{\infty}\right)\right)=D\left(y,\left(a_{i}^{\infty}\right)\right)<\frac{\varepsilon}{2}
$$

for arbitrary $y=\left(y_{1}, y_{2}, y_{3}, \ldots, y_{m}, \ldots\right) \in \bigcup_{n \geq k}\left(f_{k} \circ f_{k+1} \circ \ldots \circ f_{n}\right)^{-1}\left(t^{\infty}\right)$.

Since $y_{1}=t=a_{i}$ by Lemma 3.18 (and therefore $\frac{y_{1}-a_{i}}{2}=0$ ), and since $y_{1} \leq y_{2} \leq y_{3} \leq \ldots$, it follows that

$$
D\left(y,\left(a_{i}^{\infty}\right)\right) \leq \sup \left\{\frac{y_{2}-a_{i}}{2^{2}}, \frac{y_{3}-a_{i}}{2^{3}}, \ldots, \frac{y_{m}-a_{i}}{2^{m}}, \frac{1}{2^{m+1}}\right\} .
$$


Let $j \in\{2,3,4, \ldots, m\}$ be arbitrary. We show that

$$
\frac{y_{j}-a_{i}}{2^{j}}<\frac{\varepsilon}{2} \text {. }
$$

First we show that for each $s \in\{2,3,4, \ldots, j\}$ there is a positive integer $\ell>n_{0}$ such that $y_{s}, y_{s-1} \in\left[a_{\ell}, b_{\ell}\right]$.

For $s=2$, the claim is true since $y_{2} \in\left[a_{i}, b_{i}\right], y_{1}=a_{i}$, and $i>k \geq n_{0}$.

If $y_{2} \notin\left\{a_{n} \mid n=1,2,3, \ldots\right\}$, then $y_{2}=y_{3}=y_{4}=\cdots$ and therefore for each $s \in\{3,4,5, \ldots, j\}, y_{s}=y_{s-1}=y_{2} \in\left[a_{i}, b_{i}\right]$.

In the rest of the proof we consider the case $y_{2}=a_{i_{0}}$ for some positive integer $i_{0}$. If $i_{0} \leq k_{m}$, then $\mu\left(i_{0}\right) \in\left\{n_{0}, \mu(1), \mu(2), \mu(3), \ldots, \mu\left(k_{m}\right)\right\}$, and therefore $\mu\left(i_{0}\right) \leq k$. Since $k<i$, it follows that $\mu\left(i_{0}\right)<i$. Therefore from $y_{2}=a_{i_{0}}>a_{i}$ it follows that $a_{i_{0}}>b_{i}$, and this contradicts the fact that $a_{i_{0}}=y_{2} \in\left[a_{i}, b_{i}\right]$. So in this case $i_{0}>k_{m} \geq n_{0}$, and the claim for $s=3$ follows, since $y_{3}, y_{2} \in\left[a_{i_{0}}, b_{i_{0}}\right]$.

If $y_{3} \notin\left\{a_{n} \mid n=1,2,3, \ldots\right\}$, then $y_{3}=y_{4}=y_{5}=\cdots$, and therefore for each $s \in\{4,5,6, \ldots, j\}, y_{s}=y_{s-1}=y_{3} \in\left[a_{i_{0}}, b_{i_{0}}\right]$.

In the rest of the proof we consider the case $y_{3}=a_{i_{1}}$ for some positive integer $i_{1}$. If $i_{1} \leq k_{m-1}$, then $\mu\left(i_{1}\right) \in\left\{n_{0}, \mu(1), \mu(2), \mu(3), \ldots, \mu\left(k_{m-1}\right)\right\}$, and therefore $\mu\left(i_{1}\right) \leq k_{m}$. Since $k_{m}<i_{0}$, it follows that $\mu\left(i_{1}\right)<i_{0}$. Therefore from $y_{3}=a_{i_{1}}>a_{i_{0}}$ it follows that $a_{i_{1}}>b_{i_{0}}$, and this contradicts the fact that $a_{i_{1}}=y_{3} \in\left[a_{i_{0}}, b_{i_{0}}\right]$. So in this case $i_{1}>k_{m-1} \geq n_{0}$, and the claim follows for $s=4$, since $y_{4}, y_{3} \in\left[a_{i_{1}}, b_{i_{1}}\right]$.

Repeating this reasoning $m$ times proves that for each $s \in\{2,3,4, \ldots, j\}$ there is a positive integer $\ell>n_{0}$ such that $y_{s}, y_{s-1} \in\left[a_{\ell}, b_{\ell}\right]$.

It follows that

$$
d\left(y_{j}, a_{i}\right) \leq d\left(y_{j}, y_{j-1}\right)+\ldots+d\left(y_{3}, y_{2}\right)+d\left(y_{2}, a_{i}\right) \leq(j-1) \frac{\varepsilon}{m}<\varepsilon,
$$

since $y_{s}, y_{s-1} \in\left[a_{\ell}, b_{\ell}\right]$ for each $s$, for some $\ell>n_{0}$. Therefore $\frac{y_{j}-a_{i}}{2^{m}}<\frac{\varepsilon}{2}$.

THEOREM 3.20. Let $f:[0,1] \rightarrow 2^{[0,1]}$ be the comb function with respect to any admissible sequence $\left\{\left(a_{n}, b_{n}\right)\right\}_{n=1}^{\infty}$. Then $\varliminf_{\longleftarrow}\{[0,1], f\}_{k=1}^{\infty}$ is a dendrite.

Proof. We show that $\lim _{\longleftarrow}\{[0,1], f\}_{k=1}^{\infty}$ is homeomorphic to the inverse limit of an inverse sequence of dendrites with monotone bonding functions, which is by [20, Theorem 10.36, p. 180] a dendrite, and therefore the inverse limit $\lim _{L}\{[0,1], f\}_{k=1}^{\infty}$ is a dendrite, too.

More specifically we prove that the inverse limit $\lim _{D_{n}}\{[0,1], f\}_{k=1}^{\infty}$ is homeomorphic to $\lim _{\longleftarrow}\left\{D_{n}, f_{n}\right\}_{n=1}^{\infty}$, where $f_{n}: D_{n+1} \rightarrow \overleftarrow{D_{n}}$ is the mapping defined in Definition 3.8 and that each $f_{n}$ is monotone.

For fixed $x=\left(x_{1}, x_{2}, x_{3}, \ldots, x_{m}, a_{n+1}^{\infty}\right), x_{m} \neq a_{n+1}$, and fixed $k$, let

$$
B_{k}(x)=\left\{\left(x_{1}, x_{2}, x_{3}, \ldots, x_{m}, a_{n+1}^{k}, t^{\infty}\right), t \in\left[a_{n+1}, b_{n+1}\right]\right\} .
$$


Then each

$$
S(x)=\bigcup_{k=1}^{\infty} B_{k}(x),
$$

is the star with the center $x$ and beams $B_{k}(x), k=1,2,3, \ldots$

Using Remark 3.6 we see that

1. $f_{n}^{-1}(x)=\{x\}$ for each $x \in D_{n} \backslash \operatorname{Cl}\left(D_{n+1} \backslash D_{n}\right)$, and

2. $f_{n}^{-1}(x)$ is the star $S(x)$ for each $x \in D_{n} \cap \operatorname{Cl}\left(D_{n+1} \backslash D_{n}\right)$.

Therefore $f_{n}: D_{n+1} \rightarrow D_{n}$ is monotone for each $n$, and by [20, Theorem 10.36, p. 180]

$$
\lim _{\longleftarrow}\left\{D_{n}, f_{n}\right\}_{n=1}^{\infty}
$$

is a dendrite.

Next we show that by

$$
F\left(x_{1}, x_{2}, x_{3}, \ldots\right)=\lim _{n \rightarrow \infty} x_{n}
$$

a homeomorphism

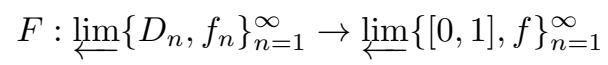

is defined.

1. First we show that $F: \lim \left\{D_{n}, f_{n}\right\}_{n=1}^{\infty} \rightarrow \underset{\lim }{\longleftarrow}\{[0,1], f\}_{n=1}^{\infty}$ is a well defined function. Take any point $\left(x_{1}, x_{2}, x_{3}, \ldots\right)$ in $\lim _{\longleftarrow}\left\{D_{n}, f_{n}\right\}_{n=1}^{\infty} \subseteq$ $\prod_{i=1}^{\infty} D_{i}$. If there is a positive integer $n_{0}$, such that $x_{n}=x_{n_{0}}$ for each $n \geq n_{0}$, then $\lim _{n \rightarrow \infty} x_{n}=x_{n_{0}}$ and $x_{n_{0}} \in D_{n_{0}} \subseteq \lim _{\longleftarrow}\{[0,1], f\}_{n=1}^{\infty}$. Therefore $F\left(x_{1}, x_{2}, x_{3}, \ldots\right) \in \lim _{\longleftarrow}\{[0,1], f\}_{n=1}^{\infty}$. If there is no such $n_{0}$, then let $i_{1}<i_{2}<i_{3}<\ldots$ be the sequence of all such integers that $x_{i_{n}} \neq x_{i_{n}+1}$ for each $n$. Then $x_{i_{n+1}}=x_{i_{n}+1} \in f_{i_{n}}^{-1}\left(x_{i_{n}}\right)$, where $f_{i_{n}}^{-1}\left(x_{i_{n}}\right)$ is the star $S\left(x_{i_{n}}\right) \subseteq D_{i_{n}+1}$ with center $x_{i_{n}}$. Therefore $x_{i_{n}}$ is of the form

$$
x_{i_{n}}=\left(y_{1}, y_{2}, y_{3}, \ldots, y_{m_{n}}, a_{i_{n}+1}^{\infty}\right) .
$$

Similarly, $x_{i_{n+1}}$ is of the form

$$
x_{i_{n+1}}=\left(z_{1}, z_{2}, z_{3}, \ldots, z_{m_{n+1}}, a_{i_{n}+2}^{\infty}\right) .
$$

Since $x_{i_{n+1}} \in S\left(x_{i_{n}}\right)$, it follows that $m_{n}<m_{n+1}$ and $y_{i}=z_{i}$ for each $i=1, \ldots, m_{n}$. From $m_{n}<m_{n+1}$ for each $n$, it follows that $m_{n} \geq n$ for each $n$. Therefore $D\left(x_{i_{n}}, x_{i_{n+1}}\right) \leq \frac{1}{2^{m_{n}}} \leq \frac{1}{2^{n}}$. It follows that the sequence $\left\{x_{n}\right\}_{n=1}^{\infty}$ is a Cauchy sequence in $\mathrm{Cl}\left(\bigcup_{n=1}^{\infty} D_{n}\right)$, and hence by Theorem 3.13 it converges to a point in $\lim _{\longleftarrow}\{[0,1], f\}_{n=1}^{\infty}$.

2. We show that $F$ is continuous. 
Take any $x=\left(x_{1}, x_{2}, x_{3}, \ldots\right) \in \lim _{\complement}\left\{D_{n}, f_{n}\right\}_{n=1}^{\infty}$ and any $\varepsilon>0$. Choose a positive integer $k$ (given by Lemma 3.19), such that

$$
\operatorname{diam}\left(\bigcup_{n \geq k}\left(f_{k} \circ f_{k+1} \circ \ldots \circ f_{n}\right)^{-1}(p)\right)<\varepsilon
$$

for each $p \in D_{k}$.

Let $B=\left\{z \in \lim _{\longleftarrow}\{[0,1], f\}_{n=1}^{\infty} \mid d(z, F(x))<\varepsilon\right\}$, and let

$$
V=P_{k}^{-1}\left(B \cap D_{k}\right) \text {, }
$$

where $P_{k}: \lim _{\longleftarrow}\left\{D_{n}, f_{n}\right\}_{n=1}^{\infty} \rightarrow D_{k}$ is the projection map to the $k$-th factor. Since $B \cap D_{k}$ is open in $D_{k}, V$ is open in $\varliminf_{\lfloor}\left\{D_{n}, f_{n}\right\}_{n=1}^{\infty}$. Since $x \in \lim _{2}\left\{D_{n}, f_{n}\right\}_{n=1}^{\infty}$ and $x_{k} \in D_{k}$, it follows from the definition of $F$ that $\overleftarrow{F}(x) \in \mathrm{Cl}\left(\bigcup_{n \geq k}\left(f_{k} \circ f_{k+1} \circ \ldots \circ f_{n}\right)^{-1}\left(x_{k}\right)\right)$. From the definition of functions $f_{j}$ it follows that $x_{k} \in \mathrm{Cl}\left(\bigcup_{n \geq k}\left(f_{k} \circ f_{k+1} \circ \ldots \circ f_{n}\right)^{-1}\left(x_{k}\right)\right)$. Therefore $d\left(x_{k}, F(x)\right)<\varepsilon$, hence $x_{k} \in B$. It follows that $x_{k} \in B \cap D_{k}$, and thus $x \in V$. Let $y=\left(y_{1}, y_{2}, y_{3}, \ldots\right) \in V$. It follows that $y_{k} \in B$, and therefore $d\left(y_{k}, F(x)\right)<\varepsilon$. Since $F(y), y_{k} \in \mathrm{Cl}\left(\bigcup_{n \geq k}\left(f_{k} \circ f_{k+1} \circ\right.\right.$ $\left.\left.\ldots \circ f_{n}\right)^{-1}\left(y_{k}\right)\right)$, it follows that $d\left(y_{k}, F(y)\right)<\varepsilon$. Hence,

$$
d(F(x), F(y)) \leq d\left(F(x), y_{k}\right)+d\left(y_{k}, F(y)\right)<2 \varepsilon .
$$

Therefore $F$ is continuous.

3. We show that $F$ is a surjection. Let

$$
y=\left(y_{1}, y_{2}, y_{3}, \ldots\right) \in \lim _{\longleftarrow}\{[0,1], f\}_{n=1}^{\infty}
$$

be arbitrarily chosen. We define a sequence $\left\{x_{n}\right\}_{n=1}^{\infty}$, such that

(a) for each $n, x_{n} \in D_{n}$,

(b) for each $n, f_{n}\left(x_{n+1}\right)=x_{n}$,

(c) $\lim _{n \rightarrow \infty} x_{n}=y$.

If $y \notin D_{n}$ for each $n$, then by Remark $3.14 y$ is of the form $y=$ $\left(a_{i_{1}}^{k_{1}}, a_{i_{2}}^{k_{2}}, a_{i_{3}}^{k_{3}}, \ldots\right)$, where for each $\ell$, it holds that $a_{i_{\ell}}<a_{i_{\ell+1}} \leq b_{i_{\ell}}$ and that $k_{\ell}$ is a positive integer. In this case we define

$$
x_{n}=\left(a_{i_{1}}^{k_{1}}, a_{i_{2}}^{k_{2}}, a_{i_{3}}^{k_{3}}, \ldots, a_{i_{m}}^{k_{m}}, a_{i_{m+1}}^{\infty}\right),
$$

where $i_{\ell} \leq n$ for each $\ell=1,2,3, \ldots, m$, and $i_{m+1}>n$. If $y \in D_{m}$ for some $m$, then define $x_{n}=y$ for $n \geq m$ and $x_{n}=\left(f_{n} \circ \cdots \circ f_{m-1}\right)(y)$ for $n<m$.

Obviously the sequence $\left\{x_{n}\right\}_{n=1}^{\infty}$ satisfies (a), (b) and (c), and therefore $F\left(x_{1}, x_{2}, x_{3}, \ldots\right)=y$.

4. Finally we show that $F$ is an injection. Let $x=\left(x_{1}, x_{2}, x_{3}, \ldots\right)$ and $y=\left(y_{1}, y_{2}, y_{3}, \ldots\right)$ be any points in $\lim _{\{}\left\{D_{n}, f_{n}\right\}_{n=1}^{\infty}$ such that $x \neq y$. Let $k$ be a positive integer such that $x_{k} \neq y_{k}$. Since $x_{k}, y_{k} \in D_{k}$, it follows that

$$
x_{k}=\left(a_{i_{1}}^{q_{1}}, a_{i_{2}}^{q_{2}}, a_{i_{3}}^{q_{3}}, \ldots, a_{i_{j}}^{q_{j}}, t^{\infty}\right)
$$


and

$$
y_{k}=\left(a_{\ell_{1}}^{r_{1}}, a_{\ell_{2}}^{r_{2}}, a_{\ell_{3}}^{r_{3}}, \ldots, a_{\ell_{m}}^{r_{m}}, s^{\infty}\right),
$$

where $i_{1}, i_{2}, \ldots, i_{j}, \ell_{1}, \ell_{2}, \ldots, \ell_{m} \leq k, t \in\left(a_{i_{j}}, b_{i_{j}}\right]$ and $s \in\left(a_{\ell_{m}}, b_{\ell_{m}}\right]$, by 1 . from Remark 3.6. Let $q=q_{1}+q_{2}+q_{3}+\ldots+q_{j}, r=r_{1}+r_{2}+$ $r_{3}+\ldots+r_{m}$. Assume that $q \leq r$. Also, let $n$ be the smallest integer such that $p_{n}\left(x_{k}\right) \neq p_{n}\left(y_{k}\right)$. If $n \leq q$ then for each $z_{1} \in \operatorname{Cl}\left(\bigcup_{i>k}\left(f_{k} \circ\right.\right.$ $\left.\left.f_{k+1} \circ \ldots \circ f_{i}\right)^{-1}\left(x_{k}\right)\right)$ and each $z_{2} \in \mathrm{Cl}\left(\bigcup_{i \geq k}\left(f_{k} \circ f_{k+1} \circ \ldots \circ f_{i}\right)^{-1}\left(y_{k}\right)\right)$ by Lemma 3.18 it follows that $p_{n}\left(z_{1}\right)=p_{n}\left(x_{k}\right)$ and $p_{n}\left(z_{2}\right)=p_{n}\left(y_{k}\right)$, and therefore

$$
D\left(z_{1}, z_{2}\right) \geq \frac{d\left(p_{n}\left(x_{k}\right), p_{n}\left(y_{k}\right)\right)}{2^{n}}
$$

Since

$$
F(x) \in \operatorname{Cl}\left(\bigcup_{i \geq k}\left(f_{k} \circ f_{k+1} \circ \ldots \circ f_{i}\right)^{-1}\left(x_{k}\right)\right)
$$

and

$$
F(y) \in \operatorname{Cl}\left(\bigcup_{i \geq k}\left(f_{k} \circ f_{k+1} \circ \ldots \circ f_{i}\right)^{-1}\left(y_{k}\right)\right)
$$

it follows that $F(x) \neq F(y)$.

If $n>q$, then $y_{k}$ is of the form

$y_{k}=\left(a_{i_{1}}^{q_{1}}, a_{i_{2}}^{q_{2}}, a_{i_{3}}^{q_{3}}, \ldots, a_{i_{j}}^{q_{j}}, a_{i_{j}}^{p}, a_{\ell_{j+1}}^{r_{j+1}}, a_{\ell_{j+2}}^{r_{j+2}}, a_{\ell_{j+3}}^{r_{j+3}}, \ldots, a_{\ell_{m}}^{r_{m}}, s^{\infty}\right)$, since $r \geq q$.

We consider several cases.

Case 1. If $p \geq 1$, then $n=q+1$, since $p_{q+1}\left(x_{k}\right)=t$ and $p_{q+1}\left(y_{k}\right)=$ $a_{i_{j}}$, and by Lemma $3.18 p_{n}(F(x))=p_{n}\left(x_{k}\right)=t \neq a_{i_{j}}=p_{n}\left(y_{k}\right)=$ $p_{n}(F(y))$, hence $F(x) \neq F(y)$.

Case 2. If $p+r_{j+1}+r_{j+2}+r_{j+3}+\ldots+r_{m}=0$, then $n=q+1$ and by Lemma $3.18 p_{n}(F(x))=p_{n}\left(x_{k}\right)=t \neq s=p_{n}\left(y_{k}\right)=p_{n}(F(y))$, hence $F(x) \neq F(y)$.

Case 3. If $p=0$ and $r_{j+1}+r_{j+2}+r_{j+3}+\ldots+r_{m}>0$ and if there is a positive integer $i \leq k$ such that $t=a_{i}$, then $F(x)=x_{k}$ and $n \leq r+1$, and it follows that $p_{n}(F(x))=p_{n}\left(x_{k}\right) \neq p_{n}\left(y_{k}\right)=p_{n}(F(y))$, where the last equality follows by Lemma 3.18 .

Case 4 . If $p=0$ and $r_{j+1}+r_{j+2}+r_{j+3}+\ldots+r_{m}>0$ and if there is a positive integer $i>k$ such that $t=a_{i}$, then $n=q+1$ since $p_{q+1}\left(y_{k}\right)=a_{l_{j+1}}$ and $l_{j+1} \leq k$, while $p_{q+1}\left(x_{k}\right)=t=a_{i}, i>k$. Therefore $p_{n}(F(x))=p_{n}\left(x_{k}\right)=a_{i} \neq a_{l_{j+1}}=p_{n}\left(y_{k}\right)=p_{n}(F(y))$, by Lemma 3.18.

Case 5. If $p=0$ and $r_{j+1}+r_{j+2}+r_{j+3}+\ldots+r_{m}>0$ and if $t \neq a_{i}$ for each positive integer $i$, then $F(x)=x_{k}$ and $n=q+1<r+1$, and we continue as in Case 3 . 
Since $F: \lim \left\{K_{n}, f_{n}\right\}_{n=1}^{\infty} \rightarrow \lim \{[0,1], f\}_{n=1}^{\infty}$ is a continuous bijection from a compact space onto a metric space, it is by [19, Theorem 5.6, p. 167] a homeomorphism.

\section{WAŻEWSKI'S UNIVERSAL DENDRITE AS AN INVERSE LIMIT WITH ONE BONDING FUNCTION}

The following example shows that the conditions of Theorem 3.20 are not sufficient to guaranty that the corresponding inverse limit is homeomorphic to Ważewski's universal dendrite.

EXAMPLE 4.1. Let for each positive integer $n,\left(a_{n}, b_{n}\right)=\left(1-\frac{1}{2^{2 n}}, 1-\right.$ $\left.\frac{1}{2^{2 n+1}}\right)$. By Theorem 3.20, $\lim _{\longleftarrow}\left\{[0,1], f_{\left(a_{n}, b_{n}\right)_{n=1}^{\infty}}\right\}_{k=1}^{\infty}$ is a dendrite. Since $a_{n}<$ $b_{n}<a_{n+1}$ for each positive integer $n$, using Lemma 4.4 and 3. from Remark 3.6 , we see that $x \in \lim \left\{[0,1], f_{\left(a_{n}, b_{n}\right)_{n=1}^{\infty}}\right\}_{k=1}^{\infty}$ is a ramification point if and only if there is a positive integer $m$, such that $x=\left(a_{m}^{\infty}\right)$. Therefore the set of all ramification points is not dense in $\lim _{\{}\left\{[0,1], f_{\left(a_{n}, b_{n}\right)_{n=1}^{\infty}}\right\}_{k=1}^{\infty}$. Hence, by Theorem 2.2, $\lim \left\{[0,1], f_{\left(a_{n}, b_{n}\right)_{n=1}^{\infty}}\right\}_{k=1}^{\infty}$ is not homeomorphic to Ważewski's universal dendrite.

In Theorem 4.5 we show that with the additional condition that the set $\left\{a_{n} \mid n=1,2,3, \ldots\right\}$ is dense in $[0,1]$, it follows that the inverse limit $\lim _{\longleftarrow}\{[0,1], f\}_{k=1}^{\infty}$ is homeomorphic to Ważewski's universal dendrite. In Theorem 4.6 we show that in fact this additional condition characterizes inverse limits $\varliminf_{\varlimsup}\{[0,1], f\}_{k=1}^{\infty}$ that are homeomorphic to Ważewski's universal dendrite.

First we prove the following lemmas.

LEMMA 4.2. Let $f:[0,1] \rightarrow 2^{[0,1]}$ be any comb function with respect to an admissible sequence $\left\{\left(a_{n}, b_{n}\right)\right\}_{n=1}^{\infty}$. Let

$$
y \in \lim _{\longleftarrow}\{[0,1], f\}_{k=1}^{\infty} \backslash \bigcup_{n=1}^{\infty} D_{n}
$$

be arbitrarily chosen. Then for each $x \in \bigcup_{n=1}^{\infty} D_{n}$ the uniquely determined arc $L$ from $x$ to $y$ satisfies the condition

$$
L \backslash\{y\} \subseteq \bigcup_{n=1}^{\infty} D_{n} .
$$

Proof. By Remark $3.14 y$ is of the form $y=\left(a_{i_{1}}^{k_{1}}, a_{i_{2}}^{k_{2}}, a_{i_{3}}^{k_{3}}, \ldots\right)$, where $a_{i_{\ell}}<a_{i_{\ell+1}} \leq b_{i_{\ell}}$ for each $\ell$. We use the same sequence $\left\{x_{n}\right\}_{n=1}^{\infty}$ as in the proof of surjectivity of $F$ in the proof of Theorem 3.20, i.e.,

$$
x_{n}=\left(a_{i_{1}}^{k_{1}}, a_{i_{2}}^{k_{2}}, a_{i_{3}}^{k_{3}}, \ldots, a_{i_{m}}^{k_{m}}, a_{i_{m+1}}^{\infty}\right) \in D_{n},
$$

where $i_{\ell} \leq n$ for each $\ell=1,2,3, \ldots, m$, and $i_{m+1}>n$. Since $D_{n+1}$ is a dendrite, there is a unique $\operatorname{arc}\left[x_{n}, x_{n+1}\right]$ from $x_{n}$ to $x_{n+1}$ in $D_{n+1}$ if $x_{n} \neq$ 
$x_{n+1}$. If $x_{n}=x_{n+1}$ let $\left[x_{n}, x_{n+1}\right]$ denote $\left\{x_{n}\right\}$. Then $A=\bigcup_{n=1}^{\infty}\left[x_{n}, x_{n+1}\right] \cup$ $\{y\}$ is an arc from $x_{1}$ to $y$, since $\left[x_{n}, x_{n+1}\right] \backslash\left\{x_{n}\right\} \in D_{n+1} \backslash D_{n}$ and since $\lim _{n \rightarrow \infty} x_{n}=y$, as shown in the above mentioned proof of Theorem 3.20. Obviously

$$
A \backslash\{y\} \subseteq \bigcup_{n=1}^{\infty} D_{n} .
$$

Next, take the unique $\operatorname{arc} B$ from $x_{1}$ to $x$ in $\bigcup_{n=1}^{\infty} D_{n}$ (the existence of such an arc follows from the fact that there is an integer $m$ such that $x_{1}, x \in D_{m}$ ). Then $\operatorname{Cl}((A \backslash B) \cup(B \backslash A))$ is an arc from $x$ to $y$ in $\lim _{\longleftarrow}\left\{[0,1], f_{\left(a_{i}, b_{i}\right)_{i=1}^{\infty}}\right\}_{k=1}^{\infty}$. Since $\lim _{\longleftarrow}\left\{[0,1], f_{\left(a_{i}, b_{i}\right)_{i=1}^{\infty}}\right\}_{k=1}^{\infty}$ is a dendrite, it follows that $\operatorname{Cl}((A \backslash B) \cup(B \backslash$ $A))=\overleftarrow{L}$. Obviously

$$
L \backslash\{y\}=\operatorname{Cl}((A \backslash B) \cup(B \backslash A)) \backslash\{y\} \subseteq \bigcup_{n=1}^{\infty} D_{n} .
$$

LEMMA 4.3. Let $f:[0,1] \rightarrow 2^{[0,1]}$ be any comb function with respect to an admissible sequence $\left\{\left(a_{n}, b_{n}\right)\right\}_{n=1}^{\infty}$. Then each

$$
y \in \lim _{\longleftarrow}\{[0,1], f\}_{k=1}^{\infty} \backslash \bigcup_{n=1}^{\infty} D_{n}
$$

is an endpoint of $\lim _{\longleftarrow}\{[0,1], f\}_{k=1}^{\infty}$ (and hence it is not a ramification point).

Proof. Assuming that $y$ is not an endpoint, using Lemma 4.2, one easily gets a simple closed curve in $\lim _{\longleftarrow}\{[0,1], f\}_{k=1}^{\infty}$. This contradicts the fact that $\lim _{\longleftarrow}\{[0,1], f\}_{k=1}^{\infty}$ is a dendrite by Theorem 3.20 .

LEMMA 4.4. Let $f:[0,1] \rightarrow 2^{[0,1]}$ be any comb function with respect to an admissible sequence $\left\{\left(a_{n}, b_{n}\right)\right\}_{n=1}^{\infty}$. Let $x \in \varliminf_{\longleftarrow}^{\lim }\{[0,1], f\}_{k=1}^{\infty}$. The following statements are equivalent.

1. $x$ is a ramification point in $\lim \{[0,1], f\}_{k=1}^{\infty}$.

2. $x$ is a ramification point in $\overleftarrow{D_{n}}$ for some positive integer $n$.

Proof. It is obvious that if there is a positive integer $n$, such that $x$ is a ramification point in $D_{n}$, then $x$ is a ramification point in $\lim \{[0,1], f\}_{k=1}^{\infty}$ (since $\left.D_{n} \subseteq \lim \{[0,1], f\}_{k=1}^{\infty}\right)$. Suppose that $x$ is a ramification point in $\lim _{\longleftarrow}\{[0,1], f\}_{k=1}^{\infty}$. Since no point of

$$
\lim _{\longleftarrow}\{[0,1], f\}_{k=1}^{\infty} \backslash \bigcup_{n=1}^{\infty} D_{n}
$$

is a ramification point in $\lim \{[0,1], f\}_{k=1}^{\infty}$, by Lemma 4.3, it follows that $x \in D_{n_{0}}$ for some positive integer $n_{0}$. Let $\left[x, x_{i}\right], i=1,2,3$, be any three arcs in $\lim _{\longleftarrow}\{[0,1], f\}_{k=1}^{\infty}$, such that $\left[x, x_{1}\right] \cup\left[x, x_{2}\right] \cup\left[x, x_{3}\right]$ is a simple triod. 
Without loss of generality we may assume that $x_{i} \in \bigcup_{n=1}^{\infty} D_{n}, i=1,2,3$, since if $x_{i} \notin \bigcup_{n=1}^{\infty} D_{n}$, we may replace $\left[x, x_{i}\right]$ by $\left[x, y_{i}\right]$, where $y_{i} \in\left(x, x_{i}\right)$, by Lemma 4.2. For each $i=1,2,3$ let $n_{i}$ be a positive integer such that $x_{i} \in D_{n_{i}}$. Let $n=\max \left\{n_{0}, n_{1}, n_{2}, n_{3}\right\}$. Obviously $\left[x, x_{1}\right] \cup\left[x, x_{2}\right] \cup\left[x, x_{3}\right]$ is a simple triod in $D_{n}$, and therefore $x$ is a ramification point in $D_{n}$.

THEOREM 4.5. Let $f:[0,1] \rightarrow 2^{[0,1]}$ be any comb function with respect to an admissible sequence $\left\{\left(a_{n}, b_{n}\right)\right\}_{n=1}^{\infty}$ such that the set $\left\{a_{n} \mid n=1,2,3, \ldots\right\}$ is dense in $[0,1]$. Then $\lim _{\longleftarrow}\{[0,1], f\}_{k=1}^{\infty}$ is homeomorphic to Ważewski's universal dendrite.

Proof. By Theorem 3.20, $D=\lim \{[0,1], f\}_{k=1}^{\infty}$ is a dendrite. We show that the set of ramification points of $D$ is dense in $D$ and that each ramification point of $D$ is of infinite order in $D$, and therefore by Theorem $2.2 D$ is homeomorphic to Ważewski's universal dendrite.

Let $y=\left(y_{1}, y_{2}, y_{3}, \ldots\right) \in D$ be arbitrarily chosen, such that $y$ is not a ramification point. We show that there is a sequence of ramification points $\left\{z_{n}\right\}_{n=1}^{\infty}$ in $D$, such that $\lim _{n \rightarrow \infty} z_{n}=y$.

If $y \in D_{n}$ for some positive integer $n$, then by 1. and 3. from Remark 3.6 (taking into account that by Lemma $4.4 y$ is not a ramification point in $D_{\ell}$ for each $\ell$ ) there are a positive integer $m$ and a real number $t \in[0,1] \backslash$ $\left\{a_{1}, a_{2}, a_{3}, \ldots\right\}$, such that

$$
y=\left(y_{1}, y_{2}, y_{3}, \ldots, y_{m-1}, t^{\infty}\right),
$$

where $y_{m-1}=a_{k}$ for some $k \leq n$, and $t \in\left(a_{k}, b_{k}\right]$. Since the set $\left\{a_{n} \mid n=\right.$ $1,2,3, \ldots\}$ is dense in $[0,1]$, there is a strictly increasing sequence $\left\{i_{n}\right\}_{n=1}^{\infty}$ of positive integers, such that $\lim _{n \rightarrow \infty} a_{i_{n}}=t$ and $a_{i_{n}} \in\left(a_{k}, b_{k}\right]$. Therefore

$$
\left\{\left(y_{1}, y_{2}, y_{3}, \ldots, y_{m-1}, a_{i_{n}}^{\infty}\right)\right\}_{n=1}^{\infty}
$$

is a sequence of ramification points in $D$, which converges to $y$.

If $y \in D \backslash \bigcup_{n=1}^{\infty} D_{n}$, then by Remark 3.14

$$
y=\left(a_{i_{1}}^{k_{1}}, a_{i_{2}}^{k_{2}}, a_{i_{3}}^{k_{3}}, \ldots\right),
$$

where for each $\ell$ it holds that $k_{\ell}>0$ and $a_{i_{\ell}}<a_{i_{\ell+1}} \leq b_{i_{\ell}}$. Then the sequence $\left\{z_{n}\right\}_{n=1}^{\infty}$, where

$$
z_{n}=\left(a_{i_{1}}^{k_{1}}, a_{i_{2}}^{k_{2}}, a_{i_{3}}^{k_{3}}, \ldots, a_{i_{n-1}}^{k_{n-1}}, a_{i_{n}}^{\infty}\right)
$$

for each $n$, is a sequence of ramification points in $D$, which converges to $y$.

Next we show that each of the ramification points is of infinite order in $D$. Let $x \in D$ be any ramification point. Then by Lemma 4.4 and 3. from Remark 3.6 there are positive integers $m$ and $j$, such that $p_{k}(x)=a_{j}$ for each positive integer $k \geq m$. Without loss of generality we may assume that $p_{k}(x) \neq a_{j}$ for each $k<m$.

Since

$$
x \in f_{j-1}^{-1}(x) \subseteq D
$$


and $f_{j-1}^{-1}(x)$ is a star with the center $x$ by Lemma 3.10 , it follows that $x$ is of infinite order in $D$.

THEOREM 4.6. Let $f:[0,1] \rightarrow 2^{[0,1]}$ be any comb function with respect to an admissible sequence $\left\{\left(a_{n}, b_{n}\right)\right\}_{n=1}^{\infty}$. Then $\lim _{\longleftarrow}\{[0,1], f\}_{k=1}^{\infty}$ is homeomorphic to Wazewski's universal dendrite if and only if the set $\left\{a_{n} \mid n=1,2,3, \ldots\right\}$ is dense in $[0,1]$.

Proof. Taking Theorem 4.5 into account it suffices to prove that if the set $\left\{a_{n} \mid n=1,2,3, \ldots\right\}$ is not dense in $[0,1]$, then $\lim _{\longleftarrow}\{[0,1], f\}_{k=1}^{\infty}$ is not homeomorphic to Ważewski's universal dendrite. If there is an interval $J=$ $\left(a_{j}, a_{k}\right) \subseteq[0,1]$ containing no $a_{n}$, let $t=\frac{a_{j}+a_{k}}{2}$ and $\delta=\frac{a_{k}-a_{j}}{2}$. For any ramification point $x$ of $\lim _{\longleftarrow}\{[0,1], f\}_{k=1}^{\infty} D\left(x,\left(t^{\infty}\right)\right) \geq \frac{d\left(p_{1}(x), t\right)}{2}>\delta$, since $p_{1}(x)=a_{n}$ for some $n$. Therefore the open ball in $\lim _{\longleftarrow}\{[0,1], f\}_{k=1}^{\infty}$ centered at $\left(t^{\infty}\right)$ with the radius $\delta$ contains no ramification points and hence by Theorem $2.2 \lim _{\longleftarrow}\{[0,1], f\}_{k=1}^{\infty}$ is not homeomorphic to Ważewski's universal dendrite.

THEOREM 4.7. There is a comb function $f$ such that $\lim _{\longleftarrow}\{[0,1], f\}_{k=1}^{\infty}$ is homeomorphic to Ważewski's universal dendrite.

Proof. Let $\left\{a_{n} \mid n \in \mathbb{N}\right\}$ be any dense subset of $(0,1)$. Inductively we define sequence $\left\{b_{n}\right\}_{n=1}^{\infty}$ in such a way that $\left\{\left(a_{n}, b_{n}\right)\right\}_{n=1}^{\infty}$ would be admissible which would by Theorem 4.5 guaranty that $\lim \{[0,1], f\}_{k=1}^{\infty}$ is homeomorphic to Ważewski's universal dendrite. For each positive integer $n$, let

$$
b_{n}=\frac{1}{2}\left(a_{n}+\min \left\{1, a_{i} \mid i<n, a_{i}>a_{n}\right\}\right) .
$$

First we show that $\lim _{n \rightarrow \infty}\left(b_{n}-a_{n}\right)=0$. Let $\varepsilon>0$ be arbitrary; choose a positive integer $k$ such that $\frac{1}{k}<\varepsilon$. For each $j \leq k$ choose $i_{j}$, such that $a_{i_{j}} \in\left(\frac{j-1}{k}, \frac{j}{k}\right)$, and let $n_{0}=\max \left\{i_{j} \mid j=1,2,3, \ldots, k\right\}$. For any $n>n_{0}$ let $a<b$ be two consecutive elements of the set $\left\{0,1, a_{i_{j}} \mid j=1,2,3, \ldots, k\right\}$ such that $a_{n} \in(a, b)$. Then $b_{n}-a_{n} \leq \frac{a_{n}+b}{2}-a_{n}=\frac{b-a_{n}}{2}<\frac{b-a}{2}<\varepsilon$.

Since for each positive integer $n$ for each $m \geq n$ it holds that if $a_{m}<a_{n}$, then $b_{m}<\frac{1}{2}\left(a_{m}+a_{n}\right)<a_{n}$, it follows that the sequence $\left\{\left(a_{n}, b_{n}\right)\right\}_{n=1}^{\infty}$ is admissible.

\section{ACKNOWLEDGEMENTS.}

This work was supported in part by the Slovenian Research Agency, under Grants P1-0285 and P1-0297.

The authors wish to thank to the anonymous referees for useful remarks and suggestions. 


\section{REFERENCES}

[1] I. Banič, On dimension of inverse limits with upper semicontinuous set-valued bonding functions, Topology Appl. 154 (2007), 2771-2778.

[2] I. Banič, Inverse limits as limits with respect to the Hausdorff metric, Bull. Austral. Math. Soc. 75 (2007), 17-22.

[3] I. Banič, Continua with kernels, Houston J. Math. 34 (2008), 145-163.

[4] I. Banič, M. Črepnjak, M. Merhar and U. Milutinović, Limits of inverse limits, Topology Appl. 157 (2010), 439-450.

[5] I. Banič, M. Črepnjak, M. Merhar and U. Milutinović, Paths through inverse limits, Topology Appl. 158 (2011), 1099-1112.

[6] I. Banič, M. Črepnjak, M. Merhar and U. Milutinović, Towards the complete classification of generalized tent maps inverse limits, Topology Appl. 160 (2013), 63-73.

[7] J. J. Charatonik, Monotone mappings of universal dendrites, Topology Appl. 38 (1991), 163-187.

[8] W. J. Charatonik and R. P. Roe, Inverse limits of continua having trivial shape, Houston J. Math. 38 (2012), 1307-1312.

[9] A. N. Cornelius, Weak crossovers and inverse limits of set-valued functions, preprint, 2009.

[10] J. Dugundji, Topology, Allyn and Bacon, Inc., Boston, 1966.

[11] S. Greenwood and J. A. Kennedy, Generic generalized inverse limits, Houston J. Math. 38 (2012), 1369-1384.

[12] A. Illanes, A circle is not the generalized inverse limit of a subset of $[0,1]^{2}$, Proc. Amer. Math. Soc. 139 (2011), 2987-2993.

[13] A. Illanes, S. B. Nadler, Jr., Hyperspaces. Fundamentals and recent advances, Marcel Dekker, Inc., New York, 1999.

[14] W. T. Ingram, W. S. Mahavier, Inverse limits of upper semi-continuous set valued functions, Houston J. Math. 32 (2006), 119-130.

[15] W. T. Ingram, Inverse limits of upper semi-continuous functions that are unions of mappings, Topology Proc. 34 (2009), 17-26.

[16] W. T. Ingram, Inverse limits with upper semi-continuous bonding functions: problems and some partial solutions, Topology Proc. 36 (2010), 353-373.

[17] K. Kuratowski, Topology. Vol. 2, Academic Press, New York-London, Warsaw, 1968.

[18] W. S. Mahavier, Inverse limits with subsets of $[0,1] \times[0,1]$, Topology Appl. 141 (2004), $225-231$.

[19] J. R. Munkres, Topology: a first course, Prentice-Hall, Inc., Englewood Cliffs, 1975.

[20] S. B. Nadler, Continuum theory. An introduction, Marcel Dekker, Inc., New York, 1992.

[21] V. Nall, Inverse limits with set valued functions, Houston J. Math. 37 (2011), 13231332 .

[22] V. Nall, Connected inverse limits with set-valued functions, Topology Proc. 40 (2012), $167-177$.

[23] V. Nall, Finite graphs that are inverse limits with a set valued function on $[0,1]$, Topology Appl. 158 (2011), 1226-1233.

[24] A. Palaez, Generalized inverse limits, Houston J. Math. 32 (2006), 1107-1119.

[25] S. Varagona, Inverse limits with upper semi-continuous bonding functions and indecomposability, Houston J. Math. 37 (2011), 1017-1034.

[26] T. Ważewski, Sour les courbes de Jordan ne renfermant aucune courbe simple fermée de Jordan, Ann. Soc. Polon. Math. 2 (1923), 49-170. 
I. Banič

Faculty of Natural Sciences and Mathematics

University of Maribor

Koroška 160, Maribor 2000

Slovenia

and

Institute of Mathematics, Physics and Mechanics

Jadranska 19, Ljubljana 1000

Slovenia

E-mail: iztok.banic@uni-mb.si

M. Črepnjak

Faculty of Natural Sciences and Mathematics

University of Maribor

Koroška 160, Maribor 2000

Slovenia

and

Faculty of Chemistry and Chemical Engineering

University of Maribor

Smetanova 17, Maribor 2000

Slovenia

E-mail: matevz.crepnjak@um.si

M. Merhar

Faculty of Natural Sciences and Mathematics

University of Maribor

Koroška 160, Maribor 2000

Slovenia

E-mail: matej.merhar@uni-mb.si

U. Milutinović

Faculty of Natural Sciences and Mathematics

University of Maribor

Koroška 160, Maribor 2000

Slovenia

and

Institute of Mathematics, Physics and Mechanics

Jadranska 19, Ljubljana 1000

Slovenia

E-mail: uros.milutinovic@uni-mb.si

T. Sovič

Faculty of Civil Engineering

University of Maribor

Smetanova 17, Maribor 2000

Slovenia

E-mail: tina.sovic@um.si

Received: 25.5.2012.

Revised: 14.12 .2012 . 